\title{
THE RADIO AND X-RAY LUMINOUS SN 2003bg AND THE CIRCUMSTELLAR DENSITY VARIATIONS AROUND RADIO SUPERNOVAE
}

\author{
A. M. Soderberg, ${ }^{1}$ R. A. Chevalier, ${ }^{2}$ S. R. Kulkarni, ${ }^{1}$ and D. A. Frail ${ }^{3}$ \\ Received 2005 December 15; accepted 2006 July 8
}

\begin{abstract}
We report extensive radio and X-ray observations of SN 2003bg, whose spectroscopic evolution shows a transition from a broad-lined Type Ic to a hydrogen-rich Type II, and later to a typical hydrogen-poor Type Ibc. We show that the extraordinarily luminous radio emission is well described by a self-absorption-dominated synchrotron spectrum, while the observed $\mathrm{X}$-ray emission at $t \approx 30$ days is adequately fit by inverse Compton scattering of the optical photons off of the synchrotron-emitting electrons. Our radio model implies a subrelativistic ejecta velocity, $\bar{v} \approx 0.24 c$, at $t_{0} \approx 10$ days after the explosion, which emphasizes that broad optical absorption lines do not imply relativistic ejecta. We find that the total energy of the radio-emitting region evolves as $E \approx 7.3 \times 10^{48}\left(t / t_{0}\right)^{0.4}$ ergs, assuming equipartition of energy between relativistic electrons and magnetic fields $\left(\epsilon_{e}=\epsilon_{B}=0.1\right)$. The circumstellar density is well described by a stellar wind profile, with modest (factor of $\sim 2$ ) episodic density enhancements that produce abrupt achromatic flux variations. We estimate an average mass-loss rate of $\dot{M} \approx 3 \times 10^{-4} M_{\odot} \mathrm{yr}^{-1}$ (assuming a wind velocity of $v_{w}=10^{3} \mathrm{~km} \mathrm{~s}^{-1}$ ) for the progenitor, consistent with the observed values for Galactic Wolf-Rayet stars. Comparison with other events reveals that $\sim 50 \%$ of radio supernovae show similar short-timescale flux variations, attributable to circumstellar density irregularities. Specifically, the radio light curves of SN 2003bg are strikingly similar to those of the Type IIb SN 2001ig, suggestive of a common progenitor evolution for these two events. Based on the relative intensity of the inferred density enhancements, we conclude that the progenitors of SNe 2003bg and 2001 ig experienced quasi-periodic mass-loss episodes just prior to the SN explosion. Finally, this study emphasizes that abrupt radio light-curve variations cannot be used as a reliable proxy for an engine-driven explosion, including off-axis gamma-ray bursts.
\end{abstract}

Subject headings: radiation mechanisms: nonthermal — radio continuum: general — supernovae: individual (SN 2003bg)

\section{INTRODUCTION}

Accounting for $\sim 10 \%$ of the nearby supernova population, Type Ibc supernovae (hereafter $\mathrm{SNe} \mathrm{Ibc}$ ) are identified by their lack of spectroscopic hydrogen and silicon features (see Filippenko 1997 for a review). Recognized as a rare subclass of core-collapse supernova $\sim 20$ years ago (Elias et al. 1985), SNe Ibc have recently enjoyed a revitalized interest. Beginning with the discovery of Type Ic SN 1998bw in temporal and spatial coincidence with gamma-ray burst (GRB) 980425 (Galama et al. 1998; Pian et al. 2000), we now know that most long-duration GRBs (e.g., Stanek et al. 2003) and X-ray flashes (XRFs; Soderberg et al. 2005b) are associated with SNe Ibc. However, radio observations of local $\mathrm{SNe}$ Ibc indicate that the inverse is not true; there is a strict limit of $\$ 10 \%$ on the fraction of SNe Ibc that could be accompanied by a GRB or XRF (Soderberg et al. 2006). The lack of hydrogen features in both SNe Ibc and GRB/XRF-associated SNe implies that they represent the explosion of massive stripped-core progenitors (Wheeler \& Levreault 1985; Woosley et al. 1999).

The popular models for $\mathrm{SNe} \mathrm{Ibc}$ progenitors include massive Wolf-Rayet (WR) stars that eject their envelopes through strong dense winds, and close binary systems where the progenitor star is stripped of its hydrogen-rich layer by the companion (Ensman $\&$ Woosley 1988). Despite dedicated archival searches, the progenitors of SNe Ibc are still poorly constrained by preexplosion

\footnotetext{
1 Division of Physics, Mathematics and Astronomy, 105-24, California Institute of Technology, Pasadena, CA 91125.

2 Department of Astronomy, University of Virginia, P.O. Box 3818, Charlottesville, VA 22903-0818.

3 National Radio Astronomy Observatory, Socorro, NM 87801.
}

images. The best photometric constraints are currently associated with SN 2002ap and SN 2004gt, which exclude WR progenitors in the top $\sim 30 \%$ and $\sim 50 \%$ of the population, respectively (Smartt et al. 2002; Gal-Yam et al. 2005; Maund et al. 2005). While intriguing, these archival observations lack the sensitivity to clearly discriminate between the WR and binary progenitor models.

By studying the circumstellar medium around the supernova, however, it is possible to place independent constraints on the progenitor. Observations show that Galactic WR stars are embedded in wind-stratified media (Chu 2002), while binary systems are associated with disrupted circumstellar media, possibly including an outflow during a common-envelope phase (Podsiadlowski et al. 1992).

Radio observations provide the most direct probe of the circumstellar density structure around supernovae (Weiler et al. 1986; Chevalier 1998). By observing the dynamical interaction of the ejecta with the surrounding medium, we are able to map out the mass-loss history of the progenitor star. The identification of irregular density profiles may therefore distinguish between WR and binary progenitor systems.

Here we present extensive radio observations of SN 2003bg, discovered as part of our ongoing radio survey of local Type Ibc supernovae. The peculiar SN 2003bg spectroscopically evolved from a broad-lined Type Ic to a hydrogen-rich Type II and later to a typical Type Ibc (M. Hamuy et al. 2006, in preparation), thereby bridging the hydrogen-rich and poor divisions of the core-collapse classification system. Our densely sampled radio light curves show that the extraordinarily luminous radio emission for a $\mathrm{SN} \mathrm{Ibc} \mathrm{is} \mathrm{characterized} \mathrm{by} \mathrm{episodic} \mathrm{short-timescale}$ 
variations. We show that these variations are well described by abrupt density enhancements in the circumstellar medium. Comparison with other radio supernovae shows that $\sim 50 \%$ of all wellstudied events similarly show evidence for abrupt light-curve variations. We review the mass-loss evolution observed (and inferred) for the progenitors of these core-collapse SNe. Using the observed radio properties for the peculiar SN 2003bg, we place constraints on the nature of its progenitor system.

The organization of this paper is as follows. Observations from the Very Large Array (VLA) and the Chandra X-ray Observatory are described in $\S 2$. Preliminary estimates of the energy, velocity, and density of the radio-emitting region are presented in $\S 3$. Modeling of the radio light curves is presented in $\S \S 4$ and 5, while a discussion of the radio polarization mechanism follows in $\S 6$. Our modeling of the X-ray emission is discussed in $\S 7$. In $\S 8$, we present a compilation of radio supernovae with abrupt lightcurve variations and review their circumstellar irregularities. Finally, in $\S 9$, we discuss the possible causes of the density enhancements surrounding SN 2003bg and the implications for the progenitor system.

\section{OBSERVATIONS}

SN 2003bg was optically discovered on 2003 February 25.7 UT, offset 16". $3 \mathrm{~W}$ and 24".6 S from the center of host galaxy MCG -05-10-15, at $d \approx 19.6 \mathrm{Mpc}$ (Wood-Vasey \& Chassagne 2003). Early spectroscopy on 2003 February 28 UT indicated that SN 2003bg was a peculiar Type Ic supernova with broad optical absorption lines, indicative of fast photospheric velocities, and comparable to those seen in SN 1998bw (Filippenko \& Chornock 2003). Soon thereafter, SN 2003bg developed strong, broad $\mathrm{H} \alpha$ emission with a P Cygni absorption component, indicating an expansion velocity of $-17,110 \mathrm{~km} \mathrm{~s}^{-1}$ (Hamuy et al. 2003). P Cygni components were also observed for $\mathrm{H} \beta$ and $\mathrm{H} \gamma$, prompting the reclassification of SN 2003bg as an unusual Type II supernova. Nebular spectra taken several months later revealed that all evidence of hydrogen had disappeared, suggesting that the SN had transitioned back to a Type Ibc event (M. Hamuy et al. 2006 , in preparation).

The peak time of the optical light curves suggests that SN 2003 bg was discovered within just a few days of explosion (M. Hamuy 2006, private communication). Throughout this paper, we therefore assume an approximate explosion date of 2003 February 22 UT.

\subsection{Very Large Array Data}

Motivated by the spectroscopic similarity of SN 2003bg to the radio-luminous SN 1998bw, we initiated radio observations of SN 2003bg on 2003 March 4.07 UT, with the Very Large Array (VLA). ${ }^{4}$ At $8.46 \mathrm{GHz}$, we detected a radio source coincident with the optical position at $\alpha(\mathrm{J} 2000.0)=04^{\mathrm{h}} 10^{\mathrm{m}} 59.42, \delta(\mathrm{J} 2000.0)=$ $-31^{\circ} 24^{\prime} 50^{\prime \prime} 3\left( \pm 00^{\prime \prime} 1\right.$ in each coordinate), with flux density of $f_{\nu}=2.51 \pm 0.05 \mathrm{mJy}$ (Fig. 1). We subsequently began an intense follow-up campaign to study the temporal and spectral evolution of the radio emission.

Radio data were collected at 1.43, 4.86, 8.46, 15.0, 22.5, and 43.3 GHz, spanning 2003 March-2005 October, and are summarized in Table 1. All VLA observations were taken in standard continuum observing mode, with a bandwidth of $2 \times 50 \mathrm{MHz}$.

\footnotetext{
4 The Very Large Array and Very Long Baseline Array are operated by the National Radio Astronomy Observatory, a facility of the National Science Foundation, operated under cooperative agreement by Associated Universities, Inc.
}

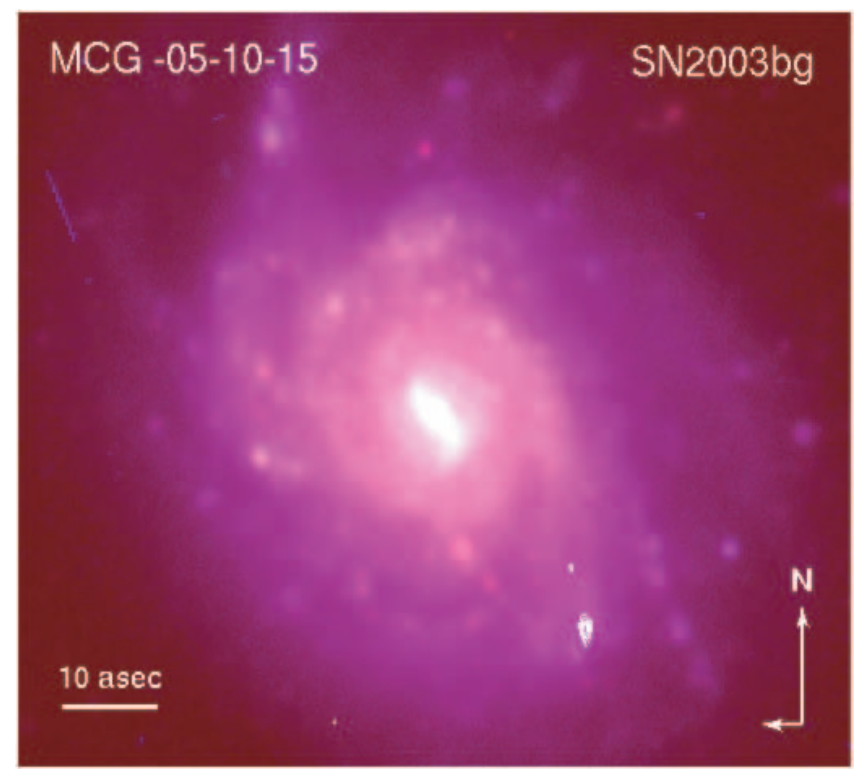

FIG. 1.-Composite color $\left(g^{\prime} r^{\prime}\right)$ image of host galaxy MCG -05-10-15, taken with the Palomar 200 inch telescope with the Large Field Camera. White contours map the radio emission from SN 2003bg, detected with the Very Large Array at $8.46 \mathrm{GHz}$ on 2005 May 31 (VLA B-array configuration). Contours are linearly spaced and correspond to flux density values between 0.5 and $4.0 \mathrm{mJy}$. The SN was located in a star-forming spiral arm of the host galaxy at position $\alpha(\mathrm{J} 2000.0)=$ $04^{\mathrm{h}} 10^{\mathrm{m}} 59^{\mathrm{s}} .42, \delta(\mathrm{J} 2000.0)=-31^{\circ} 24^{\prime} 50^{\prime \prime} .3\left( \pm 0^{\prime \prime} .1\right.$ in each coordinate $)$. We note that no diffuse emission from the host galaxy was detected in any of our radio observations.

At 22.5 and $43.3 \mathrm{GHz}$, we included referenced pointing scans to correct for the systematic $10^{\prime \prime}-20^{\prime \prime}$ pointing errors of the VLA antennas. We used 3C 48, 3C 147, and 3C 286 (J0137+331, $\mathrm{J} 0542+498$, and $\mathrm{J} 1331+305$ ) for flux calibration, while phase referencing was performed against calibrators J0407-330 and J0453-281. Data were reduced using standard packages within the Astronomical Image Processing System (AIPS). No diffuse radio emission from the host galaxy was detected in any of our observations. Flux density measurements were obtained by fitting a Gaussian model to the SN. In addition to the rms noise in each measurement, we include a systematic uncertainty of $2 \%$, due to the uncertainty in the absolute flux calibration.

The SN 2003bg radio light curves are compiled in Figures 2 and 3 , spanning $\sim 10$ to $\sim 1000$ days after the explosion. The radio evolution of SN $2003 \mathrm{bg}$ is characterized by several achromatic short-timescale $(\delta t / t \lesssim 1)$ variations. As will be discussed in $\S 8$, light-curve variations have also been observed for $\sim 50 \%$ of well-sampled radio supernovae, including Type IIL SN 1979C (Weiler et al. 1991), Type Ic SN 1998bw (Kulkarni et al. 1998; Weiler et al. 2001), and more recently for Type IIb SN 2001ig (Ryder et al. 2004).

\subsubsection{Radio Spectrum}

Shown in Figure 4 are the spectral indices between each of the adjacent radio frequencies. A spectral turnover is clearly observed for the lower frequencies at early time; at later times, the spectral slope converges to $\beta \approx-1.1$. The observed spectral shape is overall consistent with a nonthermal synchrotron spectrum, with a significant absorption component that suppresses the low-frequency emission. In the case of radio supernovae, a low-frequency turnover may be attributed to internal synchrotron self-absorption (SSA; Chevalier 1998) or free-free absorption (FFA; Weiler et al. 1986) from the external medium. The observed spectral index of the optically thick spectrum enables us 
TABLE 1

Vla Radio Flux Density Measurements of SN 2003bg

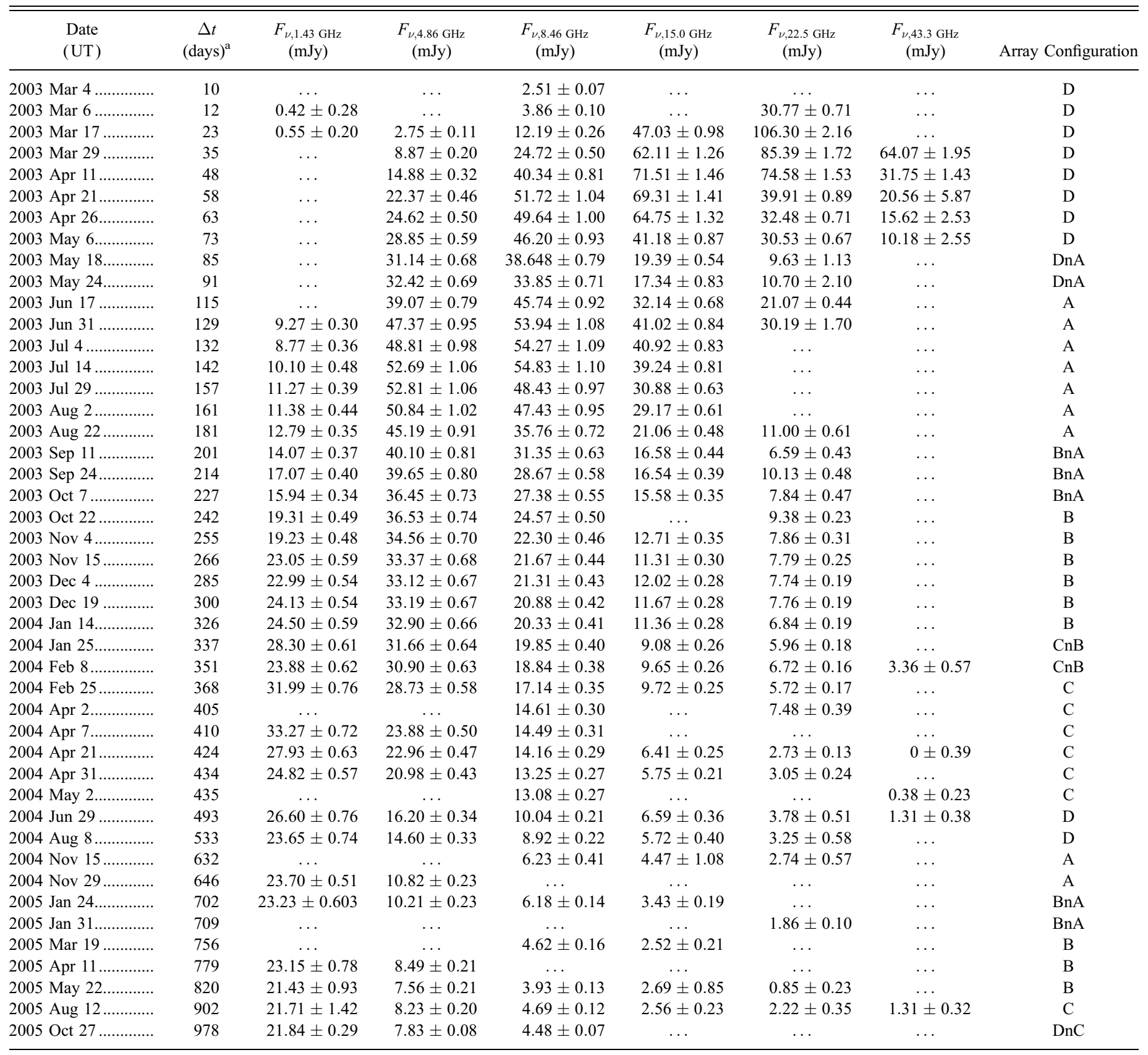

${ }^{a}$ Days since explosion, assuming an explosion date of 2003 February 22 UT.

to distinguish between these two processes. For radio supernovae dominated by SSA, the radio spectrum is approximated by

$$
F_{\nu}=1.582 F_{\nu_{p}}\left(\nu / \nu_{p}\right)^{5 / 2}\left\{1-\exp \left[-\left(\nu / \nu_{p}\right)^{-\left(5-2 \beta_{\text {thin }}\right) / 2}\right]\right\}
$$

where $\nu_{p}$ is the peak spectral frequency, $F_{\nu_{p}}$ is the flux density at $\nu_{p}$, and $\beta_{\text {thin }}$ is the optically thin spectral index (Chevalier 1998). On the other hand, for FFA dominated emission, the optically thick radio spectrum steepens to

$$
F_{\nu}=K_{1}\left(\frac{\nu}{5 \mathrm{GHz}}\right)^{\beta_{\text {thin }}} \exp \left[-K_{2}\left(\frac{\nu}{5 \mathrm{GHz}}\right)^{-2.1}\right] \mathrm{mJy}
$$

where $K_{1}$ and $K_{2}$ are normalization constants (Weiler et al. 1986).

We fit these functions to two well-sampled radio spectra, collected on 2003 March 17 and 2004 February $8(t \approx 23$ and 351 days). As shown in Figure $5,{ }^{5}$ the optically thick spectral component in each epoch is best described with a spectral index of $\beta \approx 2.5$, and thus is entirely consistent with the index predicted by the SSA dominated model. Specifically, we find the bestfit SSA parameters $F_{\nu_{p}} \approx 110 \mathrm{mJy}(50 \mathrm{mJy})$ and $\nu_{p} \approx 25 \mathrm{GHz}$ $\left(2.2 \mathrm{GHz}\right.$ ) with $\chi_{r}^{2} \approx 1.5$ (4.5) for the early (late) spectrum. The FFA-dominated models produce steeper optically thick spectra $(\beta>2.5)$ and thus provide a significantly worse fit to the data.

\footnotetext{
5 To view P. Chandra 2005, Ph.D. thesis referenced in Fig. 5 legend, see: http://www.tifr.res.in/ poonam.
} 


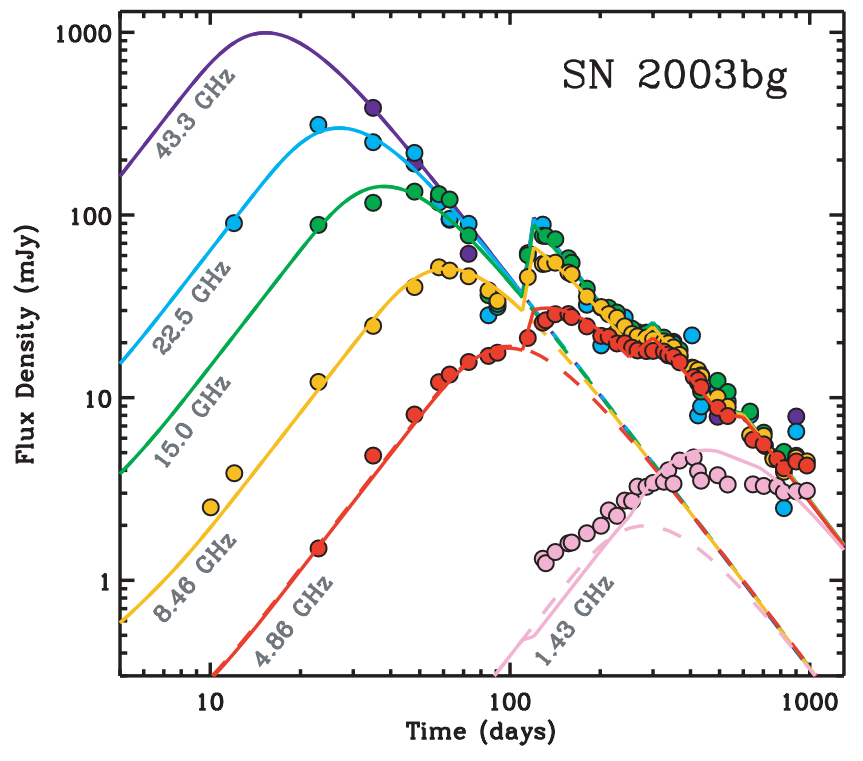

FIG. 2.- Radio light curves of SN $2003 \mathrm{bg}$. From bottom to top, the measurements were taken with the VLA at $1.43 \mathrm{GHz}$ (pink), $4.86 \mathrm{GHz}$ (red), $8.46 \mathrm{GHz}$ (orange), $15.0 \mathrm{GHz}$ (green), $22.5 \mathrm{GHz}$ (blue), and $43.3 \mathrm{GHz}$ (purple), between 2003 March and 2005 October UT $(t \sim 10-1000$ days after the explosion $)$. The data have been scaled to the $8.46 \mathrm{GHz}$ measurements by $\nu^{\beta}$, where $\beta \approx-1.1$ is the observed optically thin spectral index. An abrupt, achromatic light-curve deviation is observed at $t \sim 120$ days, and additional, weaker deviations are suggested at $t \sim 300$ and 600 days. The SSA model described in $\S 5$ is overplotted (solid lines). An extrapolation of the early fit ( $t \lesssim 110$ days) is shown for comparison (dashed lines).

We find the following best-fit FFA parameters: $K_{1} \approx 550$ (35) and $K_{2} \approx 11(0.12)$ with $\chi_{r}^{2} \approx 180(8.2)$ for the early (late) spectra. Here we have adopted $\beta_{\text {thin }} \approx-1.1$ for both models, consistent with the observed optically thin spectral indices from $t \sim 10$ to 1000 days (Fig. 4). Clearly, the SSA model fit is preferred over the FFA fit in both epochs. We conclude that internal SSA dominates the absorption on the timescale probed by the radio data.

\subsubsection{Radio Polarization of $S N 2003 \mathrm{bg}$}

Since synchrotron emission is inherently polarized, the magnetic field geometry of radio SNe can be directly probed through polarization measurements. In order to measure the polarization for SN 2003bg, we carried out a full polarization calibration of our $8.46 \mathrm{GHz}$ VLA observations, taken on 2003 October 7.4 UT $(t \approx 227$ days). This run was chosen for polarization calibration based on the high dynamic range of the radio image. To correct for the instrumental polarization, we observed calibrator J0403+ 260 over a wide range in parallactic angle and computed the leakage terms with AIPS task PCAL. We used 3C 147 to calibrate the absolute polarization angle.

We do not find any significant polarization for SN 2003bg. In particular, we place a limit on the linear polarization intensity $\left[I_{\mathrm{pol}}=\left(Q^{2}+I^{2}\right)^{1 / 2}\right.$, where $Q$ and $I$ are the Stokes parameters $]$ of $I_{\text {pol }}<0.071 \mathrm{mJy}$, corresponding to a fractional polarization limit of $\lesssim 0.8 \%(3 \sigma)$. In comparison with other radio SN linear polarization measurements, this is one of the deepest limits obtained to date: a factor of $\sim 4$ deeper than the limit for SN $1993 \mathrm{~J}(\$ 3.3 \%$; Bietenholz et al. 2003), and comparable to that for SN 1979C ( $\lesssim 1 \%$; Weiler et al. 1982).

\subsection{X-Ray Observations with Chandra}

SN 2003bg was observed with the Chandra ACIS-S detector on 2003 March 24.5 UT ( $t \sim 30$ days after the explosion) for $50.5 \mathrm{ks}$.
Pooley \& Lewin (2003) reported the detection of an X-ray source at $\alpha(\mathrm{J} 2000.0)=04^{\mathrm{h}} 10^{\mathrm{m}} 59^{\mathrm{s}} .42, \delta(\mathrm{J} 2000.0)=-31^{\circ} 24^{\prime} 50^{\prime \prime} .3\left( \pm 0^{\prime \prime} .5\right.$ in each coordinate), coincident with the optical SN position. The source was reobserved with ACIS-S on 2003 June 22.3 UT $(t \sim 120$ days) for $40.5 \mathrm{ks}$ to search for variability between the two epochs.

We retrieved the SN 2003bg data from the Chandra data archive $^{6}$ and reduced them following the CIAO science threads. ${ }^{7}$ The source is clearly detected in both epochs, and we note that no diffuse emission is detected from the host galaxy. For spectral extraction of the $\mathrm{SN}$ emission, we adopt a source aperture of 4 .'92 and a large background region, located $\sim 20^{\prime \prime}$ from the source. After subtracting the background emission, we measure count rates of $0.0117 \pm 0.0005$ and $0.0020 \pm 0.0003 \mathrm{cps}(0.3-10 \mathrm{keV})$ in the first and second epochs, respectively.

Pooley \& Lewin (2003) report that the first epoch data can be reasonably fit with several different spectral models and propose a MEKAL hot plasma model for the best fit. Given that the small number of counts prevents the model fits from being distinguished, we fit the extracted SN spectra with only two basic models, absorbed power-law and thermal bremsstrahlung. Table 2 lists the parameter values for our resulting spectral fits, where we have adopted both fixed and variable $N_{\mathrm{H}}$ values for comparison. Schlegel et al. (1998) dust maps give $E(B-V)=0.022 \mathrm{mag}$ for the position of SN 2003bg. Using the standard conversion of Predehl \& Schmitt (1995), we find $N_{\mathrm{H}}=1.3 \times 10^{20} \mathrm{~cm}^{-2}$ for the Galactic column density along our line-of-sight. The relatively few X-ray counts enable satisfactory fits $\left(\chi_{r}^{2} \sim 1\right)$ for each of the models.

Given the significantly fewer counts in the second epoch, we fit the spectrum with a fixed (Galactic) absorption. Moreover, for the power-law fit, we adopt the best-fit spectral index from our first epoch. The resulting flux values appear in Table 2 for both power-law and thermal bremsstrahlung fits. As in the case of the first epoch, both models provide an equally good representation of the data.

In Figure 6, we show the absorbed power-law model fits for epochs 1 and 2, assuming a fixed Galactic $N_{\mathrm{H}}$. Using the associated spectral parameters in Table 2, we find unabsorbed flux values of $F_{\mathrm{X}} \approx(9.3 \pm 0.4) \times 10^{-14}$ and $(1.2 \pm 0.4) \times 10^{-14} \mathrm{ergs} \mathrm{cm}^{-2} \mathrm{~s}^{-1}$ $(0.3-10 \mathrm{keV})$ for these fits, respectively.

The observed temporal evolution is $F_{\mathrm{X}} \propto t^{\alpha_{\mathrm{X}}}$ with $\alpha_{\mathrm{X}} \approx$ $-1.5 \pm-0.3$, and the implied spectral indices between the optically thin radio and X-ray band are nearly constant at $\beta_{\mathrm{RX}} \approx$ -0.94 and 0.89 for the first and second Chandra epochs, respectively. At a distance of $19.6 \mathrm{Mpc}$, the observed X-ray luminosity values are thus $L_{\mathrm{X}} \approx(4.3 \pm 0.2) \times 10^{39}$ and $(5.5 \pm$ 1.9) $\times 10^{38} \mathrm{ergs} \mathrm{s}^{-1}$, placing SN $2003 \mathrm{bg}$ among the most X-ray luminous SNe ever detected, and a factor of $\sim 10$ fainter than SN 1998bw on a comparable timescale.

\section{PRELIMINARY CONSTRAINTS}

Readhead (1994) showed that there is an upper limit to the brightness temperature, $T_{B}$, for SSA-dominated radio sources. This limit corresponds to the scenario in which the fractions of postshock energy density in relativistic electrons $\left(\epsilon_{e}\right)$ and magnetic fields $\left(\epsilon_{B}\right)$ are in equipartition, and the shocked electrons are accelerated into a power-law distribution, $N \propto \gamma^{-p}$, above a minimum Lorentz factor, $\gamma_{m}$. Under these assumptions, the equipartition brightness temperature, $T_{\mathrm{ep}} \equiv c^{2} f_{\nu_{p}} /\left(2 \pi k \theta_{\mathrm{ep}}^{2} \nu_{p}^{2}\right) \approx 5 \times 10^{10} \mathrm{~K}$, defines the

\footnotetext{
6 See: http://cda.harvard.edu/chaser/.

7 See: http://cxc.harvard.edu/ciao/threads/.
} 


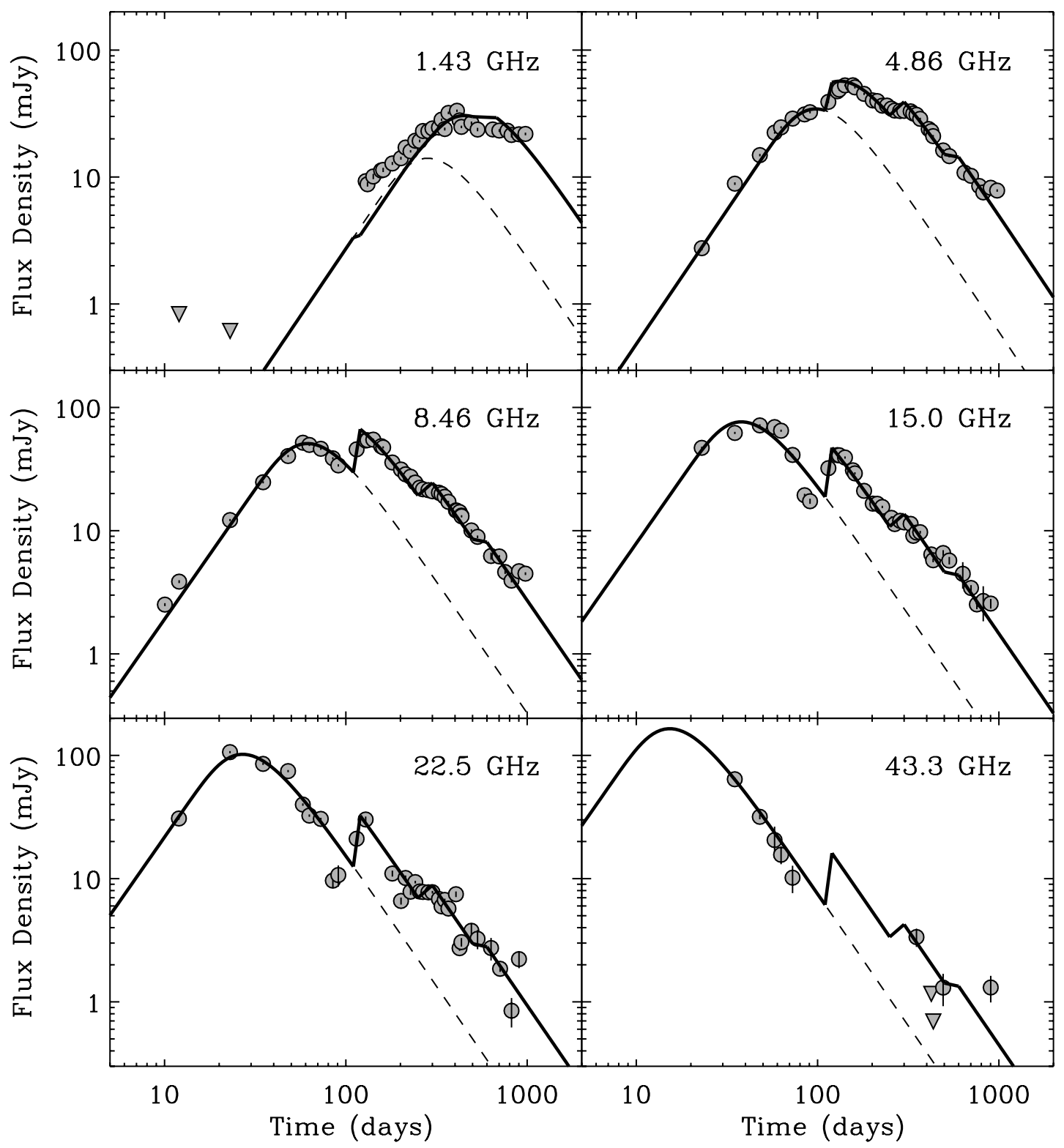

FIG. 3.- Radio light curves of SN 2003bg were taken with the VLA at frequencies 1.43, 4.86, 8.46, 15.0, 22.5, and 43.3 GHz between 2003 March and 2005 October UT. The SSA model fits described in $\S 5$ are overplotted (solid line). An extrapolation of our SSA model with no density variations is shown for comparison (dashed line)

upper bound on $T_{B}$ (Readhead 1994; Kulkarni et al. 1998). Here, $\theta_{\mathrm{ep}}$ is the equipartition size (radius) of the radio source. It is noted that $T_{\text {ep }}$ depends only weakly on the observed peak frequency and flux density.

Under the assumption that $T_{B}=T_{\text {ep }}$, we can estimate the radius and energy of the radio-emitting material for SN 2003bg. Our first VLA epoch in which the spectral turnover is observed is 2003 March 29 ( $t \sim 35$ days). In this epoch, we observed a peak flux density of $f_{\nu_{p}} \approx 85 \mathrm{mJy}$ at $\nu_{p} \approx 22.5 \mathrm{GHz}$ and an optically thin spectral index of $\beta=-(p-1) / 2 \approx-1.1$ (Fig. 4). As shown in Figure 5, on the timescale probed by our observations, the SN 2003 bg radio spectrum is well fit with a SSA model, and we therefore identify the spectral peak as the SSA frequency, $\nu_{a}$ (defined as the frequency where the optical depth due to SSA is unity; $\left.\tau_{\mathrm{SSA}}=1\right)$. Using equations (2) and (3) of Soderberg et al. (2005a, hereafter S05), the observed source properties $\left(f_{\nu_{p}}, \nu_{p}, \beta\right.$, and $d)$ imply an equipartition radius of $\theta_{\mathrm{ep}} \approx 40 \mu$ as $\left(r_{\mathrm{ep}} \approx\right.$ $1.2 \times 10^{16} \mathrm{~cm}$ ) and a total energy for the radio emitting material of $E_{\mathrm{ep}} \approx 1.7 \times 10^{48} \mathrm{ergs}$, where we have assumed $\epsilon_{e}=\epsilon_{B}=0.1$.
The average velocity of the radio shell is therefore roughly $\bar{v}_{\mathrm{ep}} \approx 0.13 c$, and the magnetic field is $B_{\mathrm{ep}} \approx 2.8 \epsilon_{B}^{1 / 2} E_{\mathrm{ep}}^{1 / 2} r_{\mathrm{ep}}^{-3 / 2} \approx$ $0.9 \mathrm{G}$.

A comparison with the ejecta velocities compiled for corecollapse SNe (Chevalier 1998) shows that SN 2003bg resembles more closely a Type Ibc (typical ejecta velocities, $\bar{v} \sim 0.1 c$ ), rather than a Type II event $(\bar{v} \sim 0.01 c)$. Moreover, the inferred ejecta velocity for SN $2003 \mathrm{bg}$ is a factor of $\sim 10$ slower than the mildly relativistic speed inferred for SN 1998bw. This analysis emphasizes that broad optical absorption lines cannot be used as a proxy for relativistic ejecta, consistent with the radio analysis for broad-lined SNe 2002ap (Berger et al. 2002) and SN 2003jd (Soderberg et al. 2006).

Our preliminary constraint on the minimum energy for SN $2003 \mathrm{bg}$ places it among the most energetic radio SNe Ibc ever observed, second only to SN 1998bw, and comparable to SN 2003L (S05). We note that additional absorption processes (e.g., FFA) and departures from equipartition (e.g., SN 1993J; Fransson \& Björnsson 1998) increase the energy budget further. 


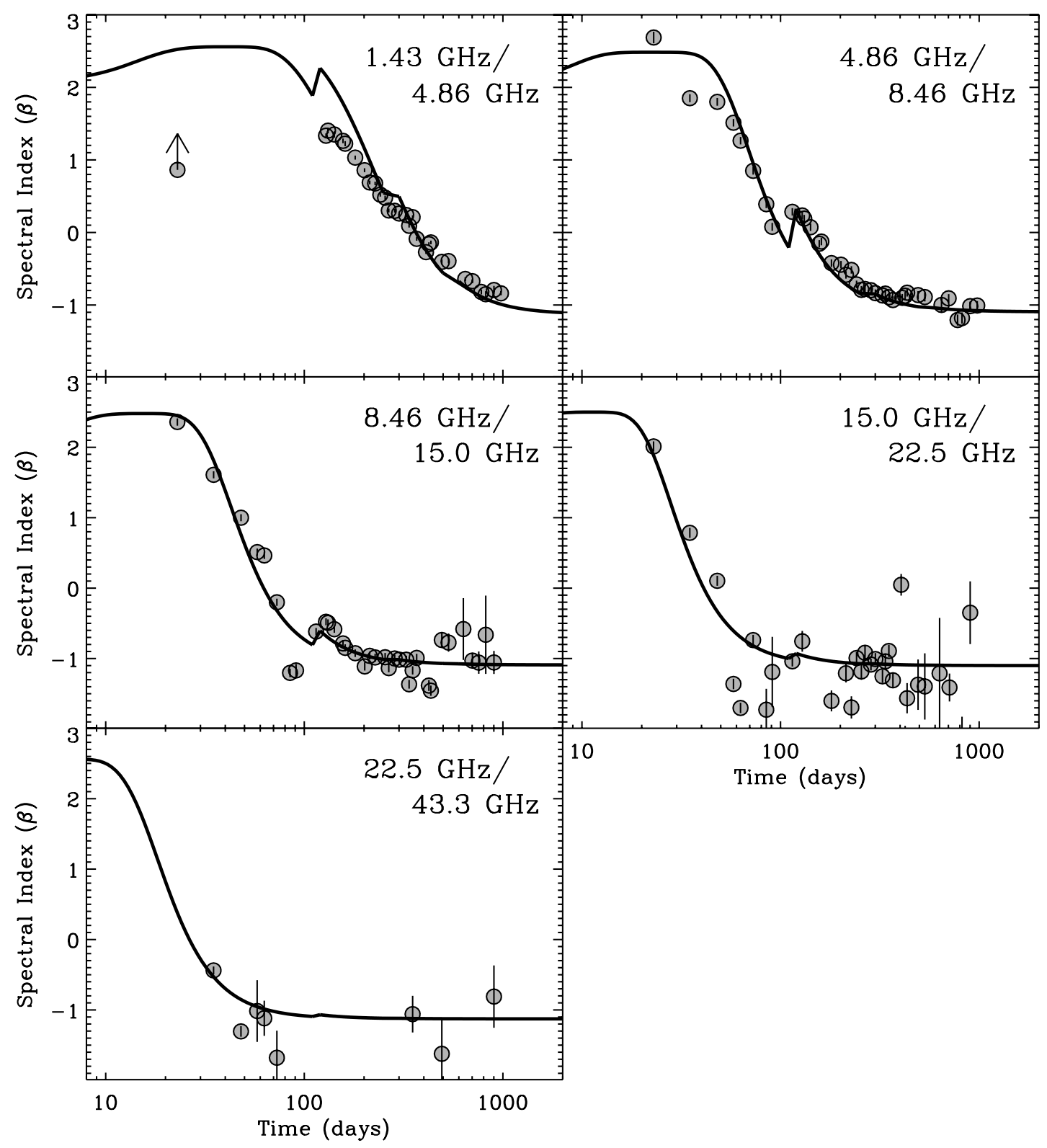

Fig. 4.- Spectral indices for the radio emission of SN 2003bg. The temporal evolution of 1.43/4.86, 4.86/8.46, 8.46/15.0, 15.0/22.5, and 22.5/43.3 GHz spectral indices are shown, along with our SSA model fits (solid lines). The abrupt variations in the spectral indices are overall consistent with our CSM density jump model ( 5 ).

Equipartition analysis may also be used to roughly constrain the characteristic synchrotron frequency, $\nu_{m}$. Equating particle kinetic energy across the shock discontinuity, S05 show that $\nu_{m} \approx$ $9.2 \times 10^{3} \epsilon_{e}^{2}(v / c)^{4} B \mathrm{GHz}$, which gives $\nu_{m} \approx 0.02 \mathrm{GHz}$ for $\epsilon_{e}=$ 0.1 , with the values of $v_{\text {ep }}$ and $B_{\text {ep }}$ given above. At $t \sim 35$ days, $\nu_{m}$ is therefore already below our radio-observing band, and the synchrotron break frequencies are ordered such that $\nu_{m}<\nu_{a}$.

Finally, we use these equipartition results at $t \approx 35$ days to estimate the mass-loss rate of the progenitor star. From Chevalier (1998), the mass-loss rate of the star, $\dot{M}$, can be derived from the postshock energy density in magnetic fields,

$$
U_{B}=\frac{B^{2}}{8 \pi} \approx \frac{\epsilon_{B}}{4 \pi}\left(\frac{\dot{M}}{v_{w}}\right) r^{-2} v^{2}
$$

where we have assumed a stellar wind-blown medium $\left(n \propto r^{-2}\right)$. We find $\dot{M} \approx 6.1 \times 10^{-5} M_{\odot} \mathrm{yr}^{-1}$ (assuming a WR wind velocity of $v_{w}=10^{3} \mathrm{~km} \mathrm{~s}^{-1}$ ).
For comparison, we estimate the mass-loss rate assuming FFA dominates the absorption. In this scenario, the shock radius is larger than the equipartition estimate, and so our equipartition parameters provide only a lower limit on the mass-loss rate of the progenitor star. Using equation (2.3) of Fransson et al. (1996), we find

$$
\frac{\dot{M}_{-5}}{v_{w, 3}}>4.3 \times 10^{3} \tau_{\mathrm{FFA}}^{1 / 2} \frac{\nu_{p}}{\mathrm{GHz}} T_{5}^{3 / 4}\left(\frac{v}{c}\right)^{3 / 2}\left(\frac{t}{35 \text { days }}\right)^{3 / 2}
$$

where $\tau_{\text {FFA }}$ is the optical depth to FFA processes, and $T_{5}$ is the temperature normalized to $10^{5} \mathrm{~K}$. Here we have adopted the notation $10^{x} Q_{x}=Q$, where $\dot{M}$ is given in units of $M_{\odot} \mathrm{yr}^{-1}$, and $v_{w}$ is given in $\mathrm{km} \mathrm{s}^{-1}$. Setting $\tau_{\mathrm{FFA}}=1$ and $T_{5}=1$, we find $\dot{M}_{-5} / v_{w, 3}>4.5 \times 10^{3}$, corresponding to a mass-loss rate of $0.045 M_{\odot} \mathrm{yr}^{-1}\left(v_{w}=10^{3} \mathrm{~km} \mathrm{~s}^{-1}\right)$ at $t \approx 35$ days. This is 3 orders of magnitude larger than that derived from the SSA interpretation. Moreover, this would imply that the total energy of the radio-emitting material (a proxy for the kinetic energy of the 


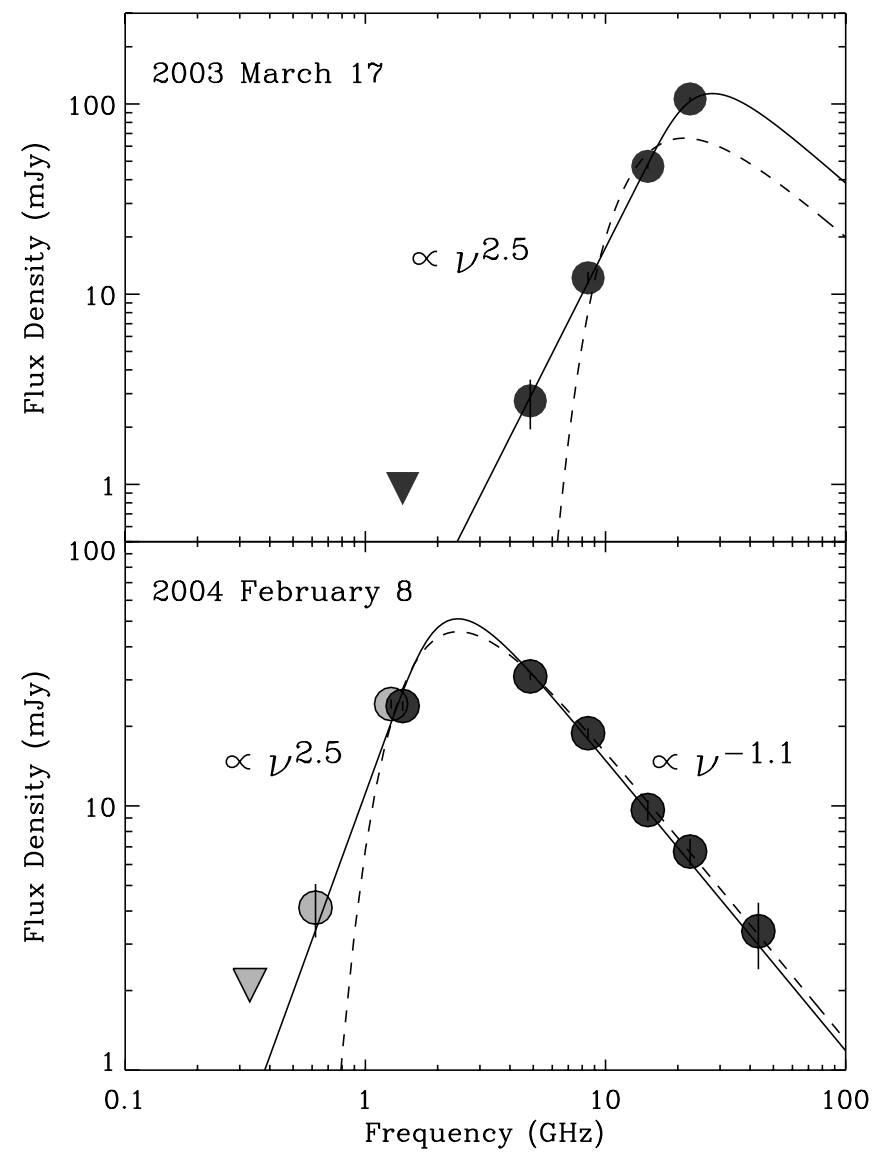

Fig. 5.-Radio spectra for SN 2003bg on 2003 Mar $17(t \approx 23$ days; top $)$ and 2004 Feb 8 UT $(t \approx 351$ days; bottom). Detections are indicated by filled circles, and upper limits as inverted triangles. In the later spectrum, we supplement our Very Large Array data (dark gray filled circles) with simultaneous observations from the Giant Meterwave Radio Telescope (GMRT; light gray circles; Chandra 2005). The low-frequency GMRT observations at $0.33,0.62$, and $1.28 \mathrm{GHz}$ confirm that the absorbed spectral index is not steeper than $\beta \approx 2.5$ (Chandra \& Ray 2005). Both spectra are best fit with a synchrotron self-absorbed spectrum (solid lines), as described in $\S 2.1 .1$. For comparison, we show best-fit FFA models (dashed lines), which are clearly inconsistent with the observed spectrum. We conclude that internal SSA dominates the observed absorption.
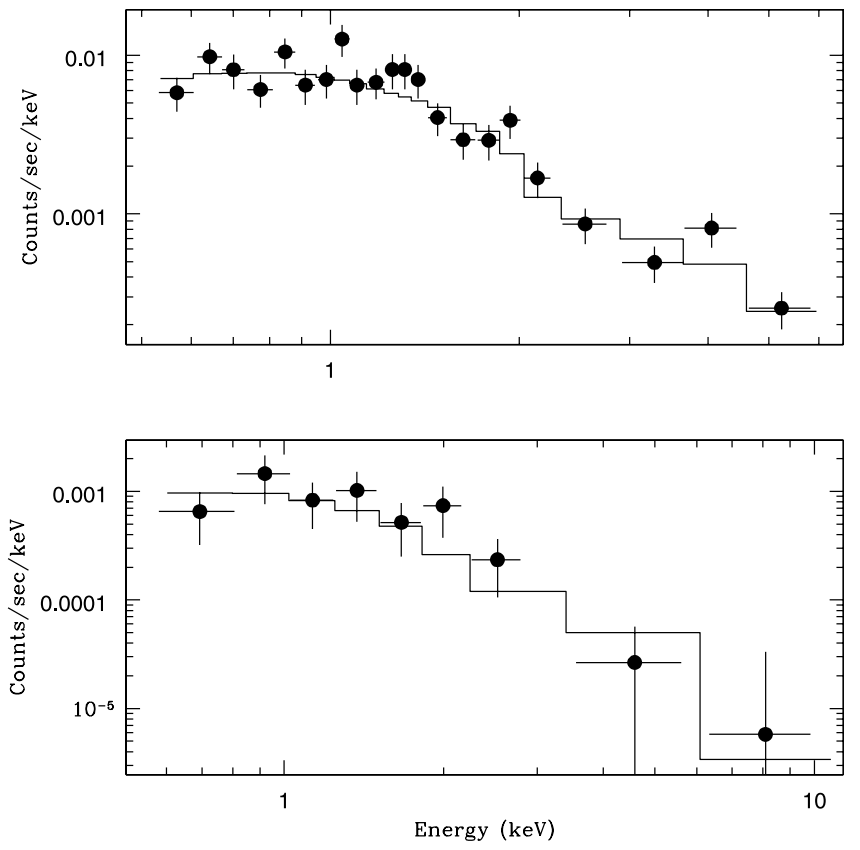

FIG. 6.-Chandra ACIS-S spectra for SN 2003bg at $t \approx 30$ days (upper panel) and at $t \approx 120$ days (bottom panel). Overplotted in both panels is an absorbed power-law fit with fixed (Galactic) $N_{\mathrm{H}}$, as described in $\S 2.2$ and Table 2 . In $\S 7$, we argue that the X-ray emission is produced by inverse Compton scattering of the optical photons in the first epoch.

fastest ejecta, $v \gtrsim 0.13 c$ ) exceeds $1.5 \times 10^{51}$ ergs. Since hydrodynamic collapse distributes the ejecta kinetic energy as $E_{K} \propto v^{-5}$ (Matzner \& McKee 1999), this would imply that the optical data (with $v \approx 0.06 c$ ) have $E_{K}>10^{53} \mathrm{ergs}$, larger than any other corecollapse SN to date. This result, taken together with the fact that a SSA model provides a significantly better fit to the radio spectra, leads us to conclude that the observed spectral turnover is due to internal SSA.

\section{SYNCHROTRON SELF-ABSORPTION MODEL}

In modeling the radio light curves of SN 2003bg, we adopt the formalism of S05, where we presented a rigorous formulation of the temporal and spectral evolution of synchrotron emission, arising from subrelativistic supernova ejecta. S05 show how the observed SN radio-emission spectrum at any single epoch is determined by three parameters: $C_{f}, C_{\tau}$, and $\nu_{m}$. Here, $C_{f}$ and $C_{\tau}$

TABLE 2

Spectral Models for Chandra ACIS-S Observations of SN 2003bg

\begin{tabular}{|c|c|c|c|c|c|c|}
\hline Model & $\begin{array}{c}N_{\mathrm{H}}^{\mathrm{a}} \\
\left(10^{22} \mathrm{~cm}^{-2}\right)\end{array}$ & $\Gamma^{\mathrm{a}}$ & $\begin{array}{c}k T^{\mathrm{a}} \\
(\mathrm{keV})\end{array}$ & $\chi^{2} /$ dof & $\begin{array}{l}\text { Flux }(0.3-10 \mathrm{keV})^{\mathrm{a}} \\
\quad\left(\operatorname{ergs~cm} \mathrm{cm}^{-2} \mathrm{~s}^{-1}\right)\end{array}$ & $\beta_{\mathrm{RX}}^{\mathrm{b}}$ \\
\hline \multicolumn{7}{|c|}{2003 March 24.5; $\Delta t=30$ days } \\
\hline \multirow[t]{2}{*}{ 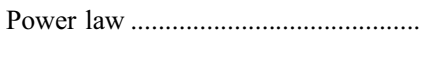 } & $0.013^{\mathrm{c}}$ & $1.7 \pm 0.2$ & $\ldots$ & $24 / 20$ & $(9.3 \pm 0.4) \times 10^{-14}$ & -0.94 \\
\hline & $0.054 \pm 0.033$ & $1.9 \pm 0.3$ & $\ldots$ & $24 / 19$ & $(9.6 \pm 0.4) \times 10^{-14}$ & -0.94 \\
\hline \multirow[t]{2}{*}{ Thermal bremsstrahlung ........................ } & $0.013^{\mathrm{c}}$ & $\ldots$ & $4.3 \pm 0.9$ & $26 / 20$ & $(7.8 \pm 0.9) \times 10^{-14}$ & -0.95 \\
\hline & $<0.018$ & $\ldots$ & $4.5 \pm 0.9$ & $25 / 19$ & $(7.7 \pm 0.9) \times 10^{-14}$ & -0.95 \\
\hline \multicolumn{7}{|c|}{2003 June $22.3 ; \Delta t=120$ days } \\
\hline 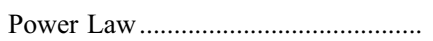 & $0.013^{\mathrm{c}}$ & 1.7 & & $5 / 7$ & $(1.2 \pm 0.4) \times 10^{-14}$ & -0.89 \\
\hline Thermal Bremsstrahlung...................... & $0.013^{\mathrm{c}}$ & $\ldots$ & $4.4 \pm 1.6$ & $4 / 7$ & $(1.2 \pm 0.2) \times 10^{-14}$ & -0.97 \\
\hline
\end{tabular}

a All errors represent $90 \%$ confidence levels.

${ }^{\mathrm{b}}$ Spectral index between optically thin radio and X-ray flux density.

${ }^{\mathrm{c}} N_{\mathrm{H}}$ fixed to the Galactic value (see $\S 2.2$ ). 

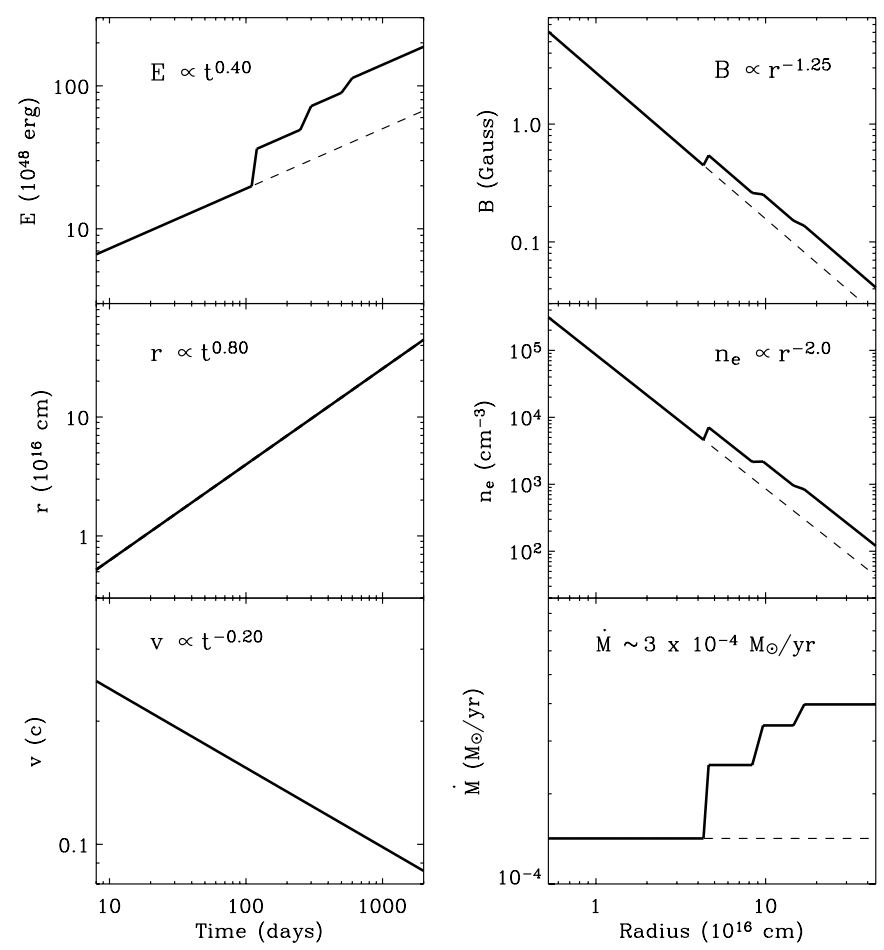

FIG. 7.- Physical parameters for SN 2003bg, based on our SSA model described in $\S 4$ and applied in $\S 5$. Left column: Temporal evolution from $t \approx 10$ to 2000 days is shown for the energy of the emitting region, shock radius, and average velocity (top to bottom). Right column: Radial profile of the magnetic field, electron number density, and mass-loss rate are shown from $r \approx 5 \times 10^{15}$ to $4 \times 10^{17} \mathrm{~cm}$. An extrapolation of our SSA model with no density variations is shown for comparison (dashed line).

are normalization constants of the flux density and optical depth, with cgs units of $\mathrm{g} \mathrm{s}^{1 / 2}$ and $\mathrm{s}^{-(2+p / 2)}$, respectively. The parameters $C_{f}, C_{\tau}$, and $\nu_{m}$ are in turn determined by the values of four physical parameters: the magnetic field, $B$, the shock radius, $r$, the minimum electron Lorentz factor, $\gamma_{m}$, and the ratio $\mathfrak{F} \equiv \epsilon_{e} / \epsilon_{B}$. With four physical parameters $\left(B, r, \gamma_{m}, \mathfrak{F}\right)$ and only three constraints $\left(C_{f}, C_{\tau}, \nu_{m, 0}\right)$, we must assume an additional constraint to find a unique solution. This constraint is obtained by adopting a value for $\mathfrak{F}$. By inverting the equations for $C_{f}, C_{\tau}$, and $\nu_{m}, \mathrm{~S} 05$ derive the following expressions for $B, r$, and $\gamma_{m}$ :

$$
\begin{array}{rl}
B= & 9.0 \times 10^{-8}(2+p)^{-6 / 17}(p-2)^{-4 / 17} \eta^{4 / 17} \mathfrak{F}^{-1 / 17} \\
& \times\left(\frac{d}{\mathrm{~cm}}\right)^{-4 / 17}\left(\frac{C_{f}}{\mathrm{~g} \mathrm{~s}^{1 / 2}}\right)^{-2 / 17} \mathrm{G} \\
& \times\left(\frac{C_{\tau}}{\mathrm{s}^{-(2+p / 2)}}\right)^{4 / 17}\left(\frac{\nu_{m}}{\mathrm{~Hz}}\right)^{-2(p-2) / 17} \\
r= & 9.3 \times 10^{12}(2+p)^{7 / 17}(p-2)^{-1 / 17} \eta^{1 / 17} \mathfrak{F}^{-1 / 17} \\
& \times\left(\frac{d}{\mathrm{~cm}}\right)^{16 / 17}\left(\frac{C_{f}}{\mathrm{~g} \mathrm{~s}^{1 / 2}}\right)^{8 / 17} C_{\tau}^{1 / 17}\left(\frac{\nu_{m}}{\mathrm{~Hz}}\right)^{-(p-2) / 34} \\
& \times\left(\frac{C^{-(2+p / 2)}}{\mathrm{s}^{-17}}\right. \\
\gamma_{m} & 2.0(2+p)^{3 / 17}(p-2)^{2 / 17} \eta^{-2 / 17} \mathfrak{F}^{2 / 17} \\
& \times\left(\frac{d}{\mathrm{~cm}}\right)^{2 / 17}\left(\frac{C_{f}}{\mathrm{~g} \mathrm{~s}^{1 / 2}}\right)^{1 / 17} \\
& \times\left(\frac{C_{\tau}}{\mathrm{s}^{-(2+p / 2)}}\right)^{-2 / 17}\left(\frac{\nu_{m}}{\mathrm{~Hz}}\right)^{(13+2 p) / 34}
\end{array}
$$

Here, $d$ is the distance to the supernova, and $\eta$ characterizes the thickness of the radiating electron shell as $r / \eta$. The number density of the synchrotron-emitting electrons then follows directly as

$$
n_{e}=\frac{p-2}{p-1} \frac{B^{2}}{8 \pi} \frac{\mathfrak{F}}{m_{e} c^{2} \gamma_{m}},
$$

where it is assumed that the contribution from electrons in a thermal distribution is negligible. The circumstellar density can be expressed in terms of a progenitor mass-loss rate

$$
\dot{M}=\frac{8 \pi}{\eta} n_{e} m_{p} r^{2} v_{w},
$$

by adopting a value for the wind velocity. The total energy of the radio-emitting material at a given epoch is then given by

$$
E=\frac{4 \pi}{\eta} r^{3} \frac{\mathfrak{F}}{\epsilon_{e}} \frac{B^{2}}{8 \pi},
$$

where it should be noted that $E$ depends not only on $\mathfrak{F}$, but also on an assumed value of $\epsilon_{e}$.

\subsection{Hydrodynamical Evolution of the Ejecta}

As discussed by Chevalier (1996), several models have been proposed for hydrodynamic evolution of the subrelativistic supernova ejecta. Based on separate assumptions, these analytic models constrain the temporal behavior of the shock radius, magnetic field, electron Lorentz factor, and energy partition fractions. Here, we adopt the standard SSA model (model 1 of Chevalier 1996) for the hydrodynamical evolution of the ejecta. This model assumes that the evolution is self-similar across the shock discontinuity, and thus $r \propto t^{\alpha_{r}}$ with $\alpha_{r}=(n-3) /(n-s)$, where $n$ characterizes the density profile of the outer SN ejecta $\left(\rho \propto r^{-n}\right)$, and $s$ characterizes the density profile of the radiating electrons within the shocked circumstellar material $\left(n_{e} \propto r^{-s}\right)$. In addition, the standard SSA model assumes that the magnetic energy density $\left(U_{B} \propto B^{2}\right)$ and the relativistic electron energy density $\left(U_{e} \propto n_{e} \gamma_{m}\right)$ scale as the total postshock energy density $(U \propto$ $\left.n_{e} v^{2}\right)$. In this scenario, the magnetic field is amplified by turbulence near the shock discontinuity, implying fixed energy fractions, $\epsilon_{e}$ and $\epsilon_{B}$, and thus a constant value of $\mathfrak{F}$ throughout the evolution of the ejecta.

The evolution of the magnetic field is determined by the CSM density profile and the expansion of the shock radius: $B \propto t^{\alpha_{B}}$, with $\alpha_{B}=\alpha_{r}(2-s) / 2-1$. We note that for a wind-stratified medium, $s=2$ and $\alpha_{B}=-1$. The minimum Lorentz factor evolves as $\gamma_{m} \propto t^{\alpha_{\gamma}}$, with $\alpha_{\gamma}=2\left(\alpha_{r}-1\right)$. From the scalings of equation (10), it follows that $E \propto t^{3 \alpha_{r}-2}$, and therefore the total postshock energy increases with time until the swept up circumstellar mass is comparable to rest mass of the SN ejecta.

\section{SSA MODEL FIT FOR SN 2003bg}

As shown in Figure 2 and briefly discussed in $\S 2$, the radio light curves for SN 2003bg are characterized by achromatic short-timescale variability, inconsistent with the strict powerlaw evolution prescribed by the standard model. In an effort to model the overall evolution of the radio ejecta, we therefore divide the multifrequency light curves into four subsections, each defined by the observed time of abrupt variation. We then apply the SSA model described in $\S 4$ to each subsection. See Figure 7 for SN2003bg based on our SSA model. 


\subsection{The Synchrotron Self-Absorption Peak}

Figure 3 shows that the first peak observed for each of the radio frequencies is chromatic, and therefore produced by a cascading spectral break. We define the time range for this chromatic evolution phase to be $t<110$ days, extending until just before the first achromatic variation is observed. As discussed in $\S 2$, the radio emission is well described by a SSA-dominated spectrum, and we therefore attribute this chromatic subsection to the passage of $\nu_{a}$ through the observed frequencies. The evolution of the spectral indices (Fig. 4) indicate that the self-absorption frequency cascades as $\nu_{a} \propto t^{-1.1}$, consistent with typical values observed for other SSA-dominated radio supernovae (Chevalier 1998).

Using our multifrequency radio data collected during the time range of the first peak, and adopting $\mathfrak{F}=1$, we fit for the constants $C_{f}$ and $C_{\tau}$, as well as the temporal indices $\alpha_{r}$ and $\alpha_{B}$. As discussed in $\S 3, \nu_{m}$ is estimated to be below the radio band during our observations and thus is unconstrained by the data. We therefore estimate $\nu_{m, 0} \approx 0.1 \mathrm{GHz}$ at our chosen reference time of $t_{0}=10$ days,${ }^{8}$ consistent with the preliminary value calculated in $\S 3$. We find a best-fit solution $\left(\chi_{r}^{2} \approx 46\right.$, dominated by interstellar scintillation) for parameters: $p \approx 3.2, C_{f} \approx 3.1 \times$ $10^{-51} \mathrm{~g} \mathrm{~s}^{1 / 2}, C_{\tau} \approx 6.2 \times 10^{38} \mathrm{~s}^{-3.6}, \alpha_{r} \approx 0.8$, and $\alpha_{B} \approx-1$. These values imply $s \approx 2, n \approx 7$, and $\alpha_{\gamma} \approx-0.4$. As explained within the Appendix of S05, we parameterize the sharpness of the $\nu_{a}$ spectral break with $\zeta=[0,1]$ and find $\zeta \approx 0.6$ for our final solution. This SSA model provides a reasonable fit to the chromatic subsection of the light curves as shown in Figures 2 and 3.

With this SSA model fit, the physical parameters of the ejecta and CSM are uniquely determined by equations (5)-(10). Chevalier (1982a) shows that for $s \approx 2$ and $n \approx 7, \eta \approx 4$. Adopting this value, we find that the expansion of the ejecta is described by $r \approx 6.2 \times 10^{15}\left(t / t_{0}\right)^{0.8} \mathrm{~cm}$, and the average velocity is $\bar{v} \approx$ $0.24\left(t / t_{0}\right)^{-0.2} c$. The radio ejecta therefore expand with a modest subrelativistic velocity, comparable to that observed for $\mathrm{SN}$ 2002ap (Berger et al. 2002), and significantly slower than SN 1998bw (bulk Lorentz factor, $\Gamma \sim 2$ on a similar timescale; Kulkarni et al. 1998; Li \& Chevalier 1999). Adopting $\epsilon_{e}=\epsilon_{B}=$ 0.1 , we find that the total energy of the radio-emitting material is given by $E \approx 7.3 \times 10^{48}\left(t / t_{0}\right)^{0.4} \mathrm{ergs}$, comparable to that of SN 1998bw. The magnetic field evolves radially as $B \approx$ $4.9\left(r / r_{0}\right)^{-1.25} \mathrm{G}$, slightly steeper than that observed for Type Ic SNe 2003L (S05), 2002ap (Berger et al. 2002), and the Type IIb SN 1993J (Fransson \& Björnsson 1998). The electron number density and associated mass-loss rate are thus given by $n_{e} \approx$ $2.2 \times 10^{5}\left(r / r_{0}\right)^{-2} \mathrm{~cm}^{-3}$ and $\dot{M} \approx 1.4 \times 10^{-4} M_{\odot} \mathrm{yr}^{-1}$, where we have assumed a WR wind velocity of $v_{w}=1000 \mathrm{~km} \mathrm{~s}^{-1}$ and adopted a nucleon-to-electron density ratio of 2 (appropriate for WR winds). This mass-loss rate is consistent with the values observed for Galactic WR stars (Cappa et al. 2004).

\subsection{Subsequent Light-Curve Variations}

Shortly after the observed SSA peak cascaded through $8.46 \mathrm{GHz}$, an abrupt rise was observed at the optically thin frequencies. By $t \sim 120$ days, the radio emission reached an achromatic second peak. Subsequent achromatic light-curve variations were later observed at $t \sim 300$ and 600 days and are most pronounced at frequencies above $\nu_{a}$. As shown in Figure 4, these variations were associated with abrupt changes in the spectral indices, most notably for those indices that straddle $\nu_{a}$.

\footnotetext{
${ }^{8}$ Here (and throughout) we use subscript " 0 " to denote the values of parameters at the reference time.
}

Possible causes for these abrupt light-curve variations include energy injection from a central engine, interaction of the reverse shock with density structures in the ejecta, and interaction of the forward shock with CSM density enhancements. Kulkarni et al. (1998) invoke energy injection to explain the achromatic second peak in the radio light curves of SN 1998bw at $t \sim 40$ days. This interpretation was supported by the inferred mildly relativistic ejecta speeds and associated prompt gamma-ray emission, suggesting that SN 1998bw was powered by a GRB-like central engine. In the case of SN 2003bg, however, the inferred ejecta speed is merely subrelativistic, and therefore there is no evidence for an engine-driven explosion. We conclude that the variations observed for SN 2003bg cannot be attributed to long-lived engine activity.

Mioduszewski et al. (2001) show that the late-time flattening observed for the SN 1993J radio light curves can be produced through the interaction of the reverse shock with density inhomogeneities within the ejecta. While the one-dimensional hydrodynamical simulations provide a reasonable fit to the smooth evolution of SN 1993J at $t \gtrsim 1000$ days, it is not clear that they could produce the abrupt rise at $t \sim 100$ days observed for SN 2003bg. Moreover, any abrupt features in the ejecta would likely be smoothed by instabilities in a three-dimensional simulation.

In the third scenario, the interaction between the forward shock and strong density variations in the circumstellar material produces abrupt changes in the temporal evolution of the radio light curves. For a wind-stratified medium with constant progenitor mass-loss rate, the circumstellar density is predicted to follow a smooth $r^{-2}$ profile. Abrupt deviations from a wind density profile have been inferred for several radio supernovae (e.g., SN 1979C; Weiler et al. 1991), and it has been argued that they are the result of a variable mass-loss history and/or the effects of a binary companion on the structure of the CSM. These densityinduced variations are more pronounced at optically thin frequencies than for optically thick. Here we show that the light-curve variations observed for SN 2003bg are similarly due to CSM density enhancements.

We model the SN 2003bg radio light curves with a simple density-enhancement model under the following assumptions: (1) the density variations can be approximated by modest abrupt jumps in the CSM, such that the density profile returns to $n_{e} \propto$ $r^{-2}$ after each enhancement; (2) the hydrodynamical evolution of the ejecta returns to the self-similar solution after each jump, thereby maintaining the same values of $\alpha_{r}, \alpha_{B}$, and $\alpha_{\gamma}$, both before and after each density enhancement; (3) the microphysics of the shock are not affected by the density jumps, and therefore $\epsilon_{e}$ and $\epsilon_{B}$ remain constant throughout the evolution of the ejecta; (4) the bulk motion of the ejecta is not significantly affected by the density jumps, since the slower, more massive material behind the shock continues to plow forward; and (5) the minimum Lorentz factor of the radiating electrons is not affected by the CSM enhancements, although the number of electrons participating in the radio emission increases.

Maintaining a self-similar evolution throughout the density variations requires that the magnetic field is enhanced by each of the CSM jumps. Moreover, since $\epsilon_{e}$ and $\epsilon_{B}$ are assumed to be fixed constants, the additional thermal energy produced by these enhancements causes an increase in the total energy of the radioemitting region. Therefore, in fitting the subsequent peaks in the SN 2003bg radio light curves, we assume that the evolution of $r$ and $\gamma_{m}$ remains effectively unchanged, while $B$ and $E$ vary according to the strength of the CSM density enhancements.

We recognize that this simple density-enhancement model is not fully consistent with the hydrodynamic evolution of the 
TABLE 3

Radio Light-Curve Peaks for SN 2003bg

\begin{tabular}{|c|c|c|c|c|c|c|c|c|}
\hline Phase & $\begin{array}{c}\Delta t \\
\text { (days) }\end{array}$ & $\begin{array}{c}r \\
\left(\times 10^{16} \mathrm{~cm}\right)\end{array}$ & $\begin{array}{c}C_{f}^{\mathrm{a}} \\
\left(\times 10^{-51} \mathrm{~g} \mathrm{~s}^{1 / 2}\right)\end{array}$ & $\begin{array}{c}C_{\tau}^{\mathrm{a}} \\
\left(\times 10^{38} \mathrm{~s}^{-3.6}\right)\end{array}$ & $\begin{array}{c}\nu_{m}{ }^{\mathrm{a}} \\
(\mathrm{GHz})\end{array}$ & $n_{e}(\text { jump })^{\mathrm{b}}$ & $B(\text { jump })^{\mathrm{b}}$ & $E$ (jump) $)^{\mathrm{b}}$ \\
\hline 1 & $\lesssim 110$ & $\lesssim 6.2$ & 3.1 & 6.2 & 0.11 & $\ldots$ & $\ldots$ & $\ldots$ \\
\hline $2 \ldots \ldots \ldots \ldots$ & $120-250$ & $6.7-12$ & 2.7 & 23 & 0.15 & 1.8 & 1.3 & 1.8 \\
\hline 3.......................... & $300-500$ & $14-21$ & 2.5 & 46 & 0.18 & 1.4 & 1.2 & 1.4 \\
\hline 4 & $\gtrsim 600$ & $\gtrsim 24$ & 2.4 & 67 & 0.19 & 1.2 & 1.1 & 1.2 \\
\hline
\end{tabular}

${ }^{\text {a }}$ Values computed at the reference time of $t_{0}=10$ days.

${ }^{\mathrm{b}}$ Factor by which the parameter is enhanced with respect to an extrapolation of the parameters from the previous phase.

ejecta. In fact, a small change in the radial expansion is expected as the shock wave reaches the higher density region. For the $n \approx 7$ self-similar solution adopted here and the modest density enhancements that we infer, we estimate this to be a minor effect $(\sim 10 \%)$. Therefore, the variations in ejecta parameters ( $B$ and $E$ ) are primarily the result of the CSM density enhancements, rather than the minor variations in radial expansion. However, the series of density jumps that we infer cause the evolution of the radio ejecta to gradually deviate from the initial radial evolution, making our model less accurate at a very late time (after several years). In addition, we note that the outflow does not immediately settle on the new self-similar solution following each density variation, but instead settles on a timescale comparable for the radius to double (i.e., the ratio of the settling time to the age of the blast wave is of order unity). Thus, the sharp features appearing in our light-curve fits are merely an artifact of our model assumptions.

As discussed in $\S 4, C_{f}, C_{\tau}$, and $\nu_{m}$ are functions of just three physical parameters: $r, \gamma_{m}$, and $B$. Of these, only $B$ responds significantly to the CSM density variations. From the Appendix of S05, we have $C_{f} \propto B^{-1 / 2}, C_{\tau} \propto B^{3+p / 2}$, and $\nu_{m} \propto B$. By fitting for $B$ at each density variation, the new values of $C_{f}, C_{\tau}$, and $\nu_{m}$ are thus directly determined.

The observed timescales and estimated circumstellar radii for each density enhancement are given in Table 3 . We note that we do not fit the short rise time of each phase, during which the ejecta are settling to the new self-similar solution. As shown in Figure 3, we find reasonable fits for each of the light-curve phases by invoking modest (factor of $\lesssim 2$ ) density enhancements. In Table 3 , we list the values of $C_{f}, C_{\tau}$, and $\nu_{m}$, and the associated enhancement factors for the density, magnetic field, and ejecta energy for each phase. It is interesting to note that the strength of the enhancements decay with time.

\section{RADIO POLARIZATION}

As discussed in $\S 2$, our VLA observations constrain the linear polarization of the radio emission to less than $\sim 0.8 \%$. Although the synchrotron emission from the radio ejecta is inherently polarized, the observed polarization level can be significantly suppressed for two reasons. First, since the SN 2003bg radiosphere is not resolved in the VLA data, our polarization limit applies to the integrated polarization of the ejecta emission. As shown for supernova remnant Cas $\mathrm{A}$, the integrated polarization can be significantly suppressed, due to the approximate circular symmetry of the radio ejecta (Rosenberg 1970). Second, the radio emission can be depolarized, due to internal Faraday dispersion within the ejecta (Burn 1966; Chevalier 1982b). For the density and magnetic field values we derive from our SSA model, we predict the integrated linear polarization of the SN 2003bg radio emission to be negligible, consistent with the observations.

\section{MODELING THE X-RAY EMISSION}

X-ray emission in supernovae can be produced by three processes (Fransson et al. 1996): (1) nonthermal synchrotron emission from radiating electrons, (2) thermal (free-free) bremsstrahlung emission from material in the circumstellar shock and/or the ejecta reverse shock, and (3) inverse Compton scattering of photospheric photons by relativistic electrons. We examine each of these scenarios in the context of the bright X-ray emission observed for SN 2003bg.

\subsection{Synchrotron Emission}

As shown in Table 2, the spectral index between the optically thin radio and $\mathrm{X}$-ray emission is $\beta \approx-0.9$ for both Chandra epochs. Assuming a constant injection spectral index from the radio to X-ray bands, we extrapolate the observed radio spectral index of $\beta \approx 1.1$ to the $X$-ray and find that the synchrotron emission underpredicts the observed X-ray flux by a factor of $\sim 10$ in both epochs. This discrepancy grows significantly larger when the synchrotron cooling break, $\nu_{c}=18 \pi m_{e} c e /\left(t^{2} \sigma_{T}^{2} B^{3}\right)$, is included, beyond which the spectrum steepens by $\Delta \beta=-0.5$. Using the magnetic field derived in $\S 5$, the cooling frequency at $t \approx 30-120$ days is $\nu_{c} \approx 6.1 \times 10^{10}\left(2.3 \times 10^{11}\right) \mathrm{Hz}$. Including the cooling break and extrapolating from $\nu_{c}$ to $\nu_{\mathrm{X}}$ as $f_{\nu} \propto \nu^{-1.6}$, the synchrotron flux falls 5 orders of magnitude below the observed X-ray emission. We therefore conclude that the X-ray emission is not dominated by synchrotron emission.

\subsection{Thermal Bremsstrahlung Emission}

In a scenario dominated by thermal bremsstrahlung processes, the X-ray emission is produced by the forward shock plowing into circumstellar material and/or the reverse shock heating of the ejecta. In this case, the strength of the X-ray emission depends strongly on the density of the CSM and unshocked ejecta. The shocked material then cools by free-free emission processes. For a wind-stratified density profile, the summed free-free luminosity from the forward and reverse shock is generalized (Chevalier \& Fransson 2003; Sutaria et al. 2003) roughly by

$$
L_{\mathrm{X}, \mathrm{FS}+\mathrm{RS}} \approx 8.6 \times 10^{34} C_{L, \mathrm{FS}+\mathrm{RS}} \dot{M}_{-5}^{2} v_{w, 3}^{-2} t_{1}^{-1} \mathrm{ergs} \mathrm{s}^{-1},
$$

where $C_{L, \mathrm{FS}+\mathrm{RS}}$ is a constant such that $C_{L, \mathrm{FS}+\mathrm{RS}}=1+(n-3) \times$ $(n-4)^{2} /(4 n-8)$. From our SSA radio model, we find $\alpha_{r} \approx 0.8$ and $\dot{M}_{-5} \approx 14\left(v_{w, 3}=1\right)$, thus $C_{L, \mathrm{FS}+\mathrm{RS}} \approx 2.8$ and $L_{\mathrm{X}, \mathrm{FS}+\mathrm{RS}} \approx$ $1.6 \times 10^{37} \mathrm{ergs} \mathrm{s}^{-1}$. The predicted X-ray luminosity is therefore a factor of $\sim 10^{2}$ fainter than the first epoch observation.

On the other hand, if we assume that the X-ray emission must be dominated by free-free processes, then this implies a significantly higher mass-loss rate of $\dot{M}_{-5} \approx 230\left(v_{w, 3}=1\right)$. However, we reiterate that a high-density model dominated by external absorption processes is inconsistent with the observed radio 
spectrum (Fig. 5). A possible compromise could be that the X-ray emission is produced by dense CSM clumps distributed with a very low filling factor $(f \ll 1)$, such that the radio emission is optically thin. This scenario was invoked for the radio-luminous Type IIn SN 1986J (Weiler et al. 1990). However, the dense CSM clumps inferred for Type IIn SNe also give rise to bright nebular $\mathrm{H} \alpha$ emission lines, which are not present in the nebular spectra of SN 2003bg. Moreover, it would be difficult to sustain the ejecta velocities inferred from our radio modeling in such a high-density environment (R. Chevalier \& C. Fransson 2006, in preparation). We conclude that the radio and optical emission do not support a scenario in which the observed X-ray flux is dominated by thermal bremsstrahlung emission.

\subsection{Inverse Compton Scattering}

X-ray emission from supernovae can also be produced by inverse Compton scattering (ICS) of the relativistic electrons by the optical photons. This process was observed to be important in explaining the observed X-ray emission for SN 2002ap (Björnsson \& Fransson 2004) and SN 2003L (S05) on timescales comparable to the optical maximum. In this scenario, the ratio of flux measured in the radio and X-ray bands, respectively, is given by

$$
\frac{F_{\text {radio }}}{F_{\mathrm{X}}} \approx \frac{U_{B}}{U_{\mathrm{ph}}},
$$

where $U_{B}$ and $U_{\mathrm{ph}}$ are the energy densities in magnetic fields and up-scattered photons, respectively. We note that equation (12) is relatively insensitive to the spectral energy index of the electrons. Björnsson \& Fransson (2004) show that

$$
U_{\mathrm{ph}} \approx 0.4 L_{\mathrm{bol}, 42}\left(t_{d} v / c\right)^{-2} \operatorname{ergs~} \mathrm{cm}^{-3},
$$

where $t_{d}$ is the time in days, $L_{\mathrm{bol}, 42}$ is the optical luminosity at $t=t_{d}$ normalized to $10^{42} \mathrm{ergs} \mathrm{s}^{-1}$, and $v$ is the shock velocity.

At the time of the first X-ray observation, $t_{d} \approx 30$, the optically thin radio flux was $F_{\text {radio }} \approx 2.2 \times 10^{-14} \mathrm{ergs} \mathrm{cm}^{-2} \mathrm{~s}^{-1}$, and the unabsorbed X-ray flux was $F_{\mathrm{X}} \approx 9.3 \times 10^{-14} \mathrm{ergs} \mathrm{cm}^{-2} \mathrm{~s}^{-1}$; therefore, $F_{\text {radio }} / F_{\mathrm{X}} \approx 0.24$. From our SSA radio modeling ( $(5)$, we estimate $B \approx 1.6 \mathrm{G}$ and $v \approx 0.19 c$ at $t \approx 30$ days. Based on the optical peak magnitude (M. Hamuy et al. 2006, private communication), we find $L_{\mathrm{bol}, 42} \approx 2.3$ at $t_{d} \approx 30$, coincident with the time of optical maximum. Therefore, $U_{B}=B^{2} / 8 \pi \approx 0.10 \mathrm{ergs} \mathrm{cm}^{-3}$, and $U_{\mathrm{ph}} \approx 0.028 \mathrm{ergs} \mathrm{cm}^{-3}$. Thus, $U_{B} / U_{\mathrm{ph}} \approx 0.28$, comparable to the observed ratio for $F_{\text {radio }} / F_{\mathrm{X}}$. We conclude that inverse Compton emission can produce the bright $\mathrm{X}$-ray luminosity in the first epoch.

The second epoch, however, cannot be explained through ICS. From our radio model at $t_{d} \approx 120, B \approx 0.41 \mathrm{G}, v \approx 0.15 c$, and $F_{\text {radio }} \approx 1.5 \times 10^{-15} \mathrm{ergs} \mathrm{cm}^{-2} \mathrm{~s}^{-1}$. The optical light curve implies $L_{\text {bol }, 42} \approx 0.23$ at this time. Therefore, we estimate $U_{B} / U_{\mathrm{ph}} \approx$ 24 and a predicted IC X-ray flux of $6.3 \times 10^{-17} \mathrm{ergs} \mathrm{cm}^{-2} \mathrm{~s}^{-1}$, which is a factor of $\sim 200$ fainter than that observed. We conclude that the X-ray flux observed at 120 days is dominated by a separate process, possibly due to a cosmic-ray-dominated SN shock that produces a late-time flattening of X-ray light curves in SNe Ibc (R. Chevalier \& C. Fransson 2006, in preparation).

\section{RADIO SUPERNOVAE AND CSM DENSITY VARIATIONS}

We have shown that the abrupt radio light-curve variations observed for SN 2003bg can be attributed to density enhance-
TABLE 4

\begin{tabular}{|c|c|c|c|c|c|}
\hline SN & Type & $\begin{array}{l}t_{\text {peak }} \\
(\mathrm{yr})\end{array}$ & $\begin{array}{c}\nu_{\text {peak }} \\
(\nu \mathrm{GHz})\end{array}$ & Variation & References $^{\mathrm{a}}$ \\
\hline 1957D......... & II & $<27$ & 4.9 & Abrupt steepening & $1,2,3$ \\
\hline $1978 \mathrm{~K} \ldots \ldots \ldots . .$. & IIn & 2.6 & 4.9 & Abrupt steepening & 4 \\
\hline 1979C ........... & IIL & 1.2 & 5.0 & Episodic bumps & $1,5,6$ \\
\hline $1980 \mathrm{~K} \ldots \ldots \ldots$ & IIL & 0.4 & 5.0 & Abrupt steepening & 1,7 \\
\hline $1986 \mathrm{~J} \ldots \ldots \ldots . . .$. & IIn & 3.8 & 4.9 & Abrupt steepening & 8 \\
\hline 1987A.......... & II & 0.0093 & 0.84 & Abrupt rise & 9,10 \\
\hline $1988 \mathrm{Z} \ldots \ldots \ldots$ & IIn & 2.5 & 4.9 & Abrupt steepening & 11 \\
\hline 1998bw....... & Ic & 0.030 & 4.9 & Episodic bumps & $12,13,14$ \\
\hline $2001 \mathrm{em} . . . . .$. & Ic/II & $<2.5$ & 4.9 & Abrupt rise & 15,16 \\
\hline 2001 ig ......... & $\mathrm{IIb}$ & 0.20 & 5.0 & Episodic bumps & 17 \\
\hline 2003 bg........ & $\mathrm{Ic} / \mathrm{II}$ & 0.27 & 4.9 & Episodic bumps & 18 \\
\hline $2004 \mathrm{C} \ldots \ldots \ldots$ & Ic & $<0.24$ & 4.9 & Abrupt rise & 19 \\
\hline $2004 \mathrm{cc} \ldots \ldots .$. & Ic & 0.068 & 8.5 & Abrupt steepening & 19 \\
\hline
\end{tabular}

Radio Supernovae with Strong Light-Curve Variations

${ }^{a}$ References.- (1) Weiler et al. 1986; (2) Cowan et al. 1994; (3) Stockdale et al. 2005; (4) Schlegel et al. 1999; (5) Weiler et al. 1992; (6) Montes et al. 2000; (7) Montes et al. 1998; (8) Bietenholz et al. 2002; (9) Ball et al. 1995; (10) Chevalier 1998; (11) Williams et al. 2002; (12) Kulkarni et al. 1998; (13) Li \& Chevalier 1999; (14) Weiler et al. 2001; (15) Stockdale et al. 2004 ; (16) Chugai \& Chevalier 2006; (17) Ryder et al. 2004; (18) this paper; (19) A. Soderberg et al. 2006, in preparation.

ments in the circumstellar medium. In comparison with wellsampled radio supernovae compiled from previous studies, ${ }^{9}$ including our ongoing survey of Type Ibc events (A. Soderberg et al. 2006 , in preparation), we find that $\sim 50 \%$ of radio supernovae similarly show evidence for abrupt achromatic variations. At the same time, there are several examples of well-studied radio supernovae with smooth light-curve evolutions, including Type Ilb SN 1993J (van Dyk et al. 1994; Bietenholz et al. 2003) and Type Ic SN 2003L (S05), among others. In Table 4, we compile the light-curve properties for radio supernovae with abrupt variability. We emphasize that this compilation contains core-collapse SNe from nearly all spectroscopic classifications, including hydrogenrich Types IIL, IIn, and IIb, in addition to hydrogen-poor Type Ibc.

Table 4 shows that the short-timescale variability observed for radio supernovae can be qualitatively divided into three types. The most common effect is an abrupt steepening of the radio light curves, as observed for SN 1988Z (Williams et al. 2002). On the other hand, an abrupt rise in the optically thin flux has been observed for a couple of events (e.g., SN 1987A; Manchester et al. 2002). More intriguingly, episodic (and perhaps periodic) lightcurve bumps are observed for SNe 1979C (Weiler et al. 1992; Montes et al. 1998, 2000), 2001ig (Ryder et al. 2004), 1998bw (Kulkarni et al. 1998; Weiler et al. 2001), and 2003bg, all of which are characterized by modest flux deviations (factor of $\lesssim 2$ ).

These three types of radio variability are generally attributed to dynamical interaction of the ejecta with irregularities in the circumstellar medium. Abrupt density variations in the media surrounding core-collapse $\mathrm{SNe}$ are known to be produced in a variety of ways, including:

1. Fluctuations in the progenitor mass-loss rate and/or wind velocity. This scenario is supported through observations of local supernova remnants and massive stars, which collectively suggest a complex picture for mass-loss during the final stage of stellar evolution.

2. Termination shocks between stellar winds ejected during the evolution of the progenitor star, which may produce abrupt density enhancements at radii of $R \gtrsim 0.1 \mathrm{pc}$ (Garcia-Segura et al.

\footnotetext{
${ }^{9}$ See: http://rsd-www.nrl.navy.mil/7213/weiler/sne-home.html.
} 
1996a, 1996b; Chevalier et al. 2004; Ramirez-Ruiz et al. 2005). Case 2 is observationally motivated by the circumstellar nebulae associated with local WR stars, which are produced by interacting stellar winds (Chu 2002).

3. A clumpy stellar wind, perhaps resulting from fragmentation of circumstellar shells by Rayleigh-Taylor instabilities (GarciaSegura et al. 1996a). This scenario is suggested by narrow $\mathrm{H} \alpha$ line profiles observed for Type IIn supernovae. In the case of SN 1988Z, Chugai \& Danziger (1994) propose a shocked dense wind component at $R \sim 5 \times 10^{16} \mathrm{~cm}$ to explain the low-velocity line emission.

4. The effects of a binary companion on the circumstellar wind profile. This case is observationally motivated by the binary companion for Type IIb SN 1993J (Maund et al. 2004) and the dense circumstellar ring around SN 1987A at $R \sim 8 \times 10^{17} \mathrm{~cm}$ (Burrows et al. 1995), commonly attributed to the effects of a companion star (Podsiadlowski 1992). In this scenario, the binary system parameters determine the circumstellar radii at which the progenitor wind is most affected.

5. A close binary progenitor system embedded in a common envelope (Podsiadlowski et al. 1992). Given that the rate of SN progenitors in common-envelope binary systems is predicted to be low ( $\sim 1 \%$ of SNe Ibc; Chugai 1997), this scenario is expected to be rare. As an example, a common-envelope system has been proposed to explain the large inferred density enhancement at $R \sim 7 \times 10^{16} \mathrm{~cm}$ responsible for strong radio (Stockdale et al. 2004), X-ray (Pooley \& Lewin 2004), and optical (H $\alpha$; Soderberg et al. 2004) emission in the unusual Type Ic SN 2001em at $t \sim 2.5$ yr (Chugai \& Chevalier 2006).

To summarize, the strength and location of circumstellar density variations depends on the method by which the density profile was disturbed. We note that cases 3 and 5 are generally associated with hydrogen-rich events, whereas cases 1,2 , and 4 are applicable to all classes of core-collapse supernovae.

Finally, in light of this compilation, it is interesting to note that an abrupt rise in the optically thin radio emission of Type Ibc supernovae can also be attributed to the presence of an off-axis gamma-ray burst jet (Paczynski 2001; Waxman 2004). As the jet sweeps up circumstellar material and decelerates, it eventually undergoes a dynamical transition to subrelativistic expansion on a timescale of $t \sim 1 \mathrm{yr}$ (Frail et al. 2000). Once subrelativistic, the jet spreads sideways, and the ejecta rapidly approach spherical symmetry. The observational signature of an off-axis GRB is therefore an abrupt optically thin rise as the jet sweeps through our line-of-sight. Due to the location of the synchrotron spectral peak, this signature is most easily detected in the radio band on a timescale of $t \sim 1-10 \mathrm{yr}$ (Soderberg et al. 2006). Yet as we have shown here, many radio supernovae show evidence for densityinduced variability on this same timescale. We therefore emphasize that abrupt flux variations in radio supernovae may not be used as a reliable proxy for an off-axis GRB jet and/or energy injection from a central engine.

\subsection{A Comparison of SN 2003bg and SN 2001ig}

A comparison of the radio light curves represented in Table 4 shows that the variations are generally unique to each supernova. However, as Figure 8 shows, the separation and intensity of the observed variations in SN 2003bg and the Type IIb SN 2001ig are strikingly similar. By simply scaling our SN 2003bg light-curve fits by a factor of $\sim 0.1$, we obtain a reasonable match to the $\mathrm{SN}$ 2001 ig radio evolution. With this scaling, we derive the following physical parameters for the SN 2001 ig radio-emitting ejecta: $r \approx 4.2 \times 10^{15}\left(t / t_{0}\right)^{0.8} \mathrm{~cm}, \bar{v} \approx 0.16 c\left(t / t_{0}\right)^{-0.2}, B \approx 3.8\left(t / t_{0}\right)^{-1} \mathrm{G}$,

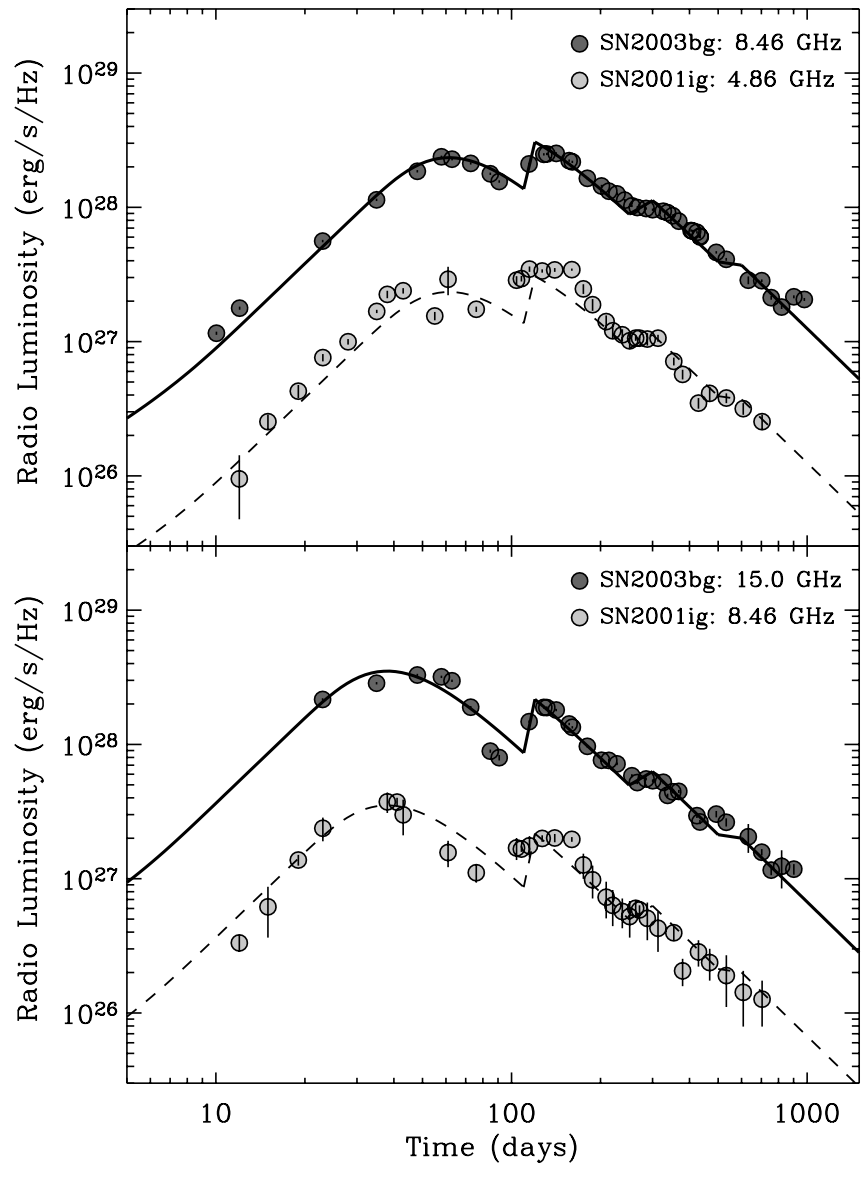

FIG. 8.- Radio light curves for SN 2003bg (dark gray filled circles) and the Type IIb SN 2001ig (light gray filled circles; Ryder et al. 2004) are compared. While SN 2003bg is overall a factor of $\sim 10$ more luminous than SN 2001ig, the shapes of the radio light curves are strikingly similar. Specifically, the SNe show evidence for light-curve variations on similar timescales, perhaps indicative of similar CSM density structures and progenitor system evolution. Our radio modeling fits (thick black lines) for SN $2003 \mathrm{bg}$ are shown at $8.46 \mathrm{GHz}$ (top panel) and $15.0 \mathrm{GHz}$ (bottom panel). By dimming the SN 2003bg fits by a factor of $\sim 10$, we obtain a suitable match to the SN 2001ig light curves.

and $E \approx 1.3 \times 10^{48}\left(t / t_{0}\right)^{0.4}$ ergs for $\epsilon_{e}=\epsilon_{B}=0.1$. The electron number density is thus given by $n_{e} \approx 3.0 \times 10^{5}\left(r / r_{0}\right)^{-2} \mathrm{~cm}^{-3}$, with successive peaks of relative intensity comparable to those observed in SN 2003bg (Table 3) and an associated mass-loss rate of $\dot{M} \approx 8.6 \times 10^{-5} M_{\odot} \mathrm{yr}^{-1}\left(v_{w}=1000 \mathrm{~km} \mathrm{~s}^{-1}\right)$. We note that this mass-loss estimate is comparable to that reported by Ryder et al. (2004), but they adopt free-free absorption for the dominant absorption process and a progenitor wind velocity of $10 \mathrm{~km} \mathrm{~s}^{-1}$, typical of red supergiant Type II SN progenitors. Our SSA modeling, however, shows that the ejecta velocity of SN 2001 ig is consistent with those of SNe Ibc, and the mass-loss rate is in fact comparable to Galactic WR stars, similar to the case of SN 2003 b.

Figure 8 shows that strong light-curve deviations are observed for both $\mathrm{SNe}$ on timescales of $\sim 120$ and 300 days, with evidence for a weaker feature at $t \sim 600$ days. Making the reasonable approximation that the ejecta evolve as $r \sim 5 \times 10^{15}\left(t / t_{0}\right)^{0.8} \mathrm{~cm}$, these variations correspond to density enhancements at radial distances of $R \sim 4 \times 10^{16}$ and $8 \times 10^{16} \mathrm{~cm}$. Assuming a progenitor wind velocity of $v_{w}=1000 \mathrm{~km} \mathrm{~s}^{-1}$, we infer ejection timescales of $\sim 12$ and 24 yr before the SN explosion. Moreover, as shown in Figure 8, the strength of the light-curve variations decreases with time, implying that the largest enhancements are closest to the explosion site. 
In addition to their radio light curves, the optical evolution for $\mathrm{SNe} 2003 \mathrm{bg}$ and $2001 \mathrm{ig}$ is also similar. In both cases there was spectroscopic evidence for a thin layer of hydrogen near the time of optical maximum (Phillips et al. 2001; Hamuy et al. 2003); however, nebular spectra revealed no hydrogen emission in either event (Filippenko \& Chornock 2002; M. Hamuy 2006, private communication). ${ }^{10}$ This evolution is characteristic of Type IIb supernovae (e.g., SN 1993J; Filippenko et al. 1994). However, their inferred ejecta velocities resemble more closely those of $\mathrm{SNe}$ Ibc and therefore imply compact (WR) progenitors. We conclude that these two events are intermediate between Types IIb and $\mathrm{Ibc}$ and show more overall similarities to hydrogen-poor events. Moreover, the likeness of their observed radio and optical evolution may indicate similar environments and progenitor evolutions.

\section{THE PROGENITORS OF SNe 2003bg AND 2001ig}

The overall resemblance of SN 2003bg and SN 2001ig as outlined in the previous section suggests that a similar process is responsible for the observed density variations. Based on the location $\left(R \gtrsim 5 \times 10^{16} \mathrm{~cm}\right)$ and relative intensity (factor of $\left.\lesssim 2\right)$ of the density enhancements, we find that cases 1 and 4 (a variable mass-loss rate and the tidal effects of a binary companion, respectively) provide the most likely explanations for the inferred circumstellar structure.

Noting the abrupt fluctuations in the SN 2001ig radio light curves, Ryder et al. (2004) recently proposed a binary-induced pinwheel nebula for the inferred episodic density enhancements. In this scenario, the colliding stellar winds produce a smooth dusty plume that spirals in the plane of the binary orbit. A highly eccentric orbit is required to produce structure at well-defined intervals, and a favorable viewing angle is necessary to reproduce the observed flux variations. Here the location and intensity of the pinwheel structure is determined by the binary parameters. Ryder et al. (2006) argue further support for this model based on the discovery of a late-B-late-F supergiant coincident (rms uncertainty of 0.03 , corresponding to $1.7 \mathrm{pc}$ at $d \approx 11.5 \mathrm{Mpc}$ ) with the SN 2001 ig optical position, which they interpret as the putative binary companion. However, in cases of observed pinwheel nebulae (e.g., Tuthill et al. 1999), the dust plumes are attributed to the strong wind-wind interaction between a WR and O or early B star companion. Taken together with the substantial uncertainty in the position of the putative companion, this suggests that a binary-induced pinwheel nebula for SN 2001ig is not clearly substantiated.

Adopting a pinwheel model for both SNe 2003bg and 2001ig would imply that the geometry of their circumstellar nebulae is nearly identical, and thus they share remarkably similar binary systems. Moreover, this model would imply that our viewing angle with respect to their circumstellar nebulae must also be very similar. While this scenario cannot be ruled out, it is clearly unlikely.

Assuming a single-star progenitor model, the mass-loss rates derived through our SSA modeling for SNe 2003bg and 2001ig imply massive WR progenitors. Broadband observations of Galactic WR stars show that the circumstellar media for these events are often significantly disturbed. In particular, X-ray observations show evidence for colliding stellar winds and dense clumps (e.g., Hillier 2003). If the progenitors of SNe 2003bg and 2001ig were single WR stars, then the observed density variations could be

\footnotetext{
${ }^{10}$ Given the similarity between the two $\mathrm{SNe}$, it is natural to question whether earlier observations of SN 2001 ig would have revealed a Type Ic spectrum, as was observed for SN 2003bg at $t \sim 6$ days past explosion.
}

produced by a series of enhanced mass-loss episodes. To be consistent with the radio data, the mass-loss ejections must ramp up as the star nears explosion. In this scenario, the observed variation timescale and the lack of CSM structure at radii inward of $R \sim 4 \times 10^{16} \mathrm{~cm}$ may be explained by a quasi-periodic mechanism that drives enhanced mass-loss episodes every $\sim 12$ yr. If accompanied by wind velocity variations, these episodes could produce shells of circumstellar material, overall consistent with the circularly symmetric density structures that we infer for $\mathrm{SNe}$ $2003 \mathrm{bg}$ and $2001 \mathrm{ig}$. The observed similarity between SNe 2003bg and $2001 \mathrm{ig}$ implies that these mass-loss episodes may be common among some fraction of hydrogen-poor events.

These clues suggest that episodic variations in the progenitor mass-loss rate and/or wind velocity provide a reasonable explanation for the radio light-curve variations observed for both SNe 2003bg and 2001ig. Given the predicted timescale for the mass ejections, these two SNe provide some of the best evidence for unusual mass-loss evolution immediately preceding the supernova explosion. While the presence of a binary companion cannot be ruled out for either of these hydrogen-poor events, additional studies of short-timescale variability in radio supernovae will enable discrimination between single and binary progenitor models.

\section{CONCLUSIONS}

We report extensive radio and X-ray observations of SN 2003bg. The spectroscopic evolution of this unusual supernova shows a transition from a broad-lined Type Ic to a hydrogen-rich Type II, and later to a typical hydrogen-poor Type Ibc. This evolution strengthens the connection between Type II and Type Ibc (including broad-lined) events. We show that the extraordinarily luminous radio emission for a SN Ibc is well described by a selfabsorption dominated synchrotron spectrum, while the observed $\mathrm{X}$-ray emission at $t \approx 30$ days is adequately fit by inverse Compton scattering of the optical photons off of the synchrotron-emitting electrons. Our radio model implies a subrelativistic ejecta velocity, $\bar{v} \approx 0.24 c$, and a size of $r \approx 6.2 \times 10^{15} \mathrm{~cm}$ at $t_{0} \approx 10$ days. This analysis emphasizes that broad optical absorption lines do not imply relativistic ejecta. We find that the total energy of the radio-emitting region evolves as $E \approx 7.3 \times 10^{48}\left(t / t_{0}\right)^{0.4} \mathrm{ergs}$, assuming equipartition of energy between relativistic electrons and magnetic fields $\left(\epsilon_{e}=\epsilon_{B}=0.1\right)$. The circumstellar density is well described by stellar wind profile, $\propto r^{-2}$, with modest (factor of 2) episodic enhancements that produce abrupt achromatic flux variations. We show that free-free absorption does not contribute significantly to the radio spectrum and estimate an average massloss rate of $\dot{M} \approx 3 \times 10^{-4} M_{\odot} \mathrm{yr}^{-1}$, consistent with observed values for local Wolf-Rayet stars.

Comparison with other events reveals that $\sim 50 \%$ of radio supernovae show similar short-timescale light-curve variations, which are attributable to circumstellar density irregularities. This compilation emphasizes that abrupt radio light-curve variations cannot be used as a reliable proxy for an engine-driven explosion, including off-axis gamma-ray bursts.

Finally, the radio light curves and spectroscopic evolution for SN 2003bg are strikingly similar to those of SN $2001 \mathrm{ig}$, suggestive of a common progenitor evolution for these two events. The overall similarity of SNe 2003bg and 2001ig to radio SNe Ibc is suggestive of a compact Wolf-Rayet progenitor model. Based on the relative intensity of the inferred density enhancements, we conclude that the progenitors of hydrogen-poor SNe 2003bg and 2001ig experienced quasi-periodic mass-loss episodes just prior to the $\mathrm{SN}$ explosion. 
We are indebted to Barry Clark for his generous scheduling of the VLA for this project. We thank M. Hamuy and A. Filippenko for making their optical SN 2003bg data available to us during this study. The authors thank Re'em Sari, Doug Leonard, Edo Berger, Michael Rupen, Paolo Mazzali, Avishay Gal-Yam, P. Brian Cameron, Derek Fox, Ehud Nakar, and Kurt Weiler for useful discussions. Caltech SN research is supported by NSF and NASA grants. A. M. S. acknowledges support by the National Radio Astronomy Observatory Graduate Summer Student Research Assistantship program and the NASA Graduate Student Research Program. R. A. C. acknowledges NSF grant AST-0307366.

Ball, L., Campbell-Wilson, D., Crawford, D. F., \& Turtle, A. J. 1995, ApJ, 453,

\section{REFERENCES}

864

Berger, E., Kulkarni, S. R., \& Chevalier, R. A. 2002, ApJ, 577, L5

Bietenholz, M. F., Bartel, N., \& Rupen, M. P. 2002, ApJ, 581, 1132 2003, ApJ, 597, 374

Björnsson, C., \& Fransson, C. 2004, ApJ, 605, 823

Burn, B. J. 1966, MNRAS, 133, 67

Burrows, C. J., et al. 1995, ApJ, 452, 680

Cappa, C., Goss, W. M., \& van der Hucht, K. A. 2004, AJ, 127, 2885

Chandra, P. 2005, Ph.D. thesis, Tata Institute of Fundamental Research, http:// www.tifr.res.in/ poonam

Chandra, P., \& Ray, A. 2005, in ASP Conf. Ser. 342, Supernovae as Cosmological Lighthouses, ed. M. Turatto, et al. (San Francisco: ASP), 430

Chevalier, R. A. 1982a, ApJ, 258, 790 . $1982 \mathrm{~b}$, ApJ, 259, 302

1996, in ASP Conf. Ser. 93, Radio Emission from the Stars and the

Sun, ed. A. R. Taylor, \& J. M. Paredes (San Francisco: ASP), 125 1998, ApJ, 499, 810

Chevalier, R. A., \& Fransson, C. 2003, Supernovae and Gamma-Ray Bursters, ed. K. Weiler (Berlin: Springer), 171

Chevalier, R. A., Li, Z.-Y., \& Fransson, C. 2004, ApJ, 606, 369

Chu, Y.-H. 2002, in ASP Conf. Ser. 260, Interacting Winds from Massive Stars, ed. A. F. J. Moffat, \& N. St-Louis (San Francisco: ASP), 109

Chugai, N. N. 1997, Astron. Rep., 41, 672

Chugai, N. N., \& Chevalier, R. A. 2006, ApJ, 641, 1051

Chugai, N. N., \& Danziger, I. J. 1994, MNRAS, 268, 173

Cowan, J. J., Roberts, D. A., \& Branch, D. 1994, ApJ, 434, 128

Elias, J. H., Matthews, K., Neugebauer, G., \& Persson, S. E. 1985, ApJ, 296, 379

Ensman, L. M., \& Woosley, S. E. 1988, ApJ, 333, 754

Filippenko, A. V. 1997, ARA\&A, 35, 309

Filippenko, A. V., \& Chornock, R. 2002, IAU Circ., 7988, 3 2003, IAU Circ., 8084, 4

Filippenko, A. V., Matheson, T., \& Barth, A. J. 1994, AJ, 108, 2220

Frail, D. A., Waxman, E., \& Kulkarni, S. R. 2000, ApJ, 537, 191

Fransson, C., \& Björnsson, C. 1998, ApJ, 509, 861

Fransson, C., Lundqvist, P., \& Chevalier, R. A. 1996, ApJ, 461, 993

Gal-Yam, A., et al. 2005, ApJ, 630, L29

Galama, T. J., et al. 1998, Nature, 395, 670

Garcia-Segura, G., Langer, N., \& Mac Low, M.-M. 1996a, A\&A, 316, 133

Garcia-Segura, G., Mac Low, M.-M., \& Langer, N. 1996b, A\&A, 305, 229

Hamuy, M., Phillips, M., \& Thomas-Osip, J. 2003, IAU Circ., 8088, 3

Hillier, D. J. 2003, in IAU Symp. 212, A Massive Star Odyssey: From Main Sequence to Supernova, ed. K. van der Hucht, A. Herrero, \& E. César (San Francisco: ASP), 70

Kulkarni, S. R., et al. 1998, Nature, 395, 663

Li, Z., \& Chevalier, R. A. 1999, ApJ, 526, 716

Manchester, R. N., et al. 2002, Publ. Astron. Soc. Australia, 19, 207

Matzner, C. D., \& McKee, C. F. 1999, ApJ, 510, 379

Maund, J. R., Smartt, S. J., Kudritzki, R. P., Podsiadlowski, P., \& Gilmore, G. F. 2004, Nature, 427, 129

Maund, J. R., Smartt, S. J., \& Schweizer, F. 2005, ApJ, 630, L33

Mioduszewski, A. J., Dwarkadas, V. V., \& Ball, L. 2001, ApJ, 562, 869

Montes, M. J., van Dyk, S. D., Weiler, K. W., Sramek, R. A., \& Panagia, N. 1998, ApJ, 506, 874
Montes, M. J., Weiler, K. W., Van Dyk, S. D., Panagia, N., Lacey, C. K., Sramek, R. A., \& Park, R. 2000, ApJ, 532, 1124

Paczynski, B. 2001, Acta Astron., 51, 1

Phillips, M. M., et al. 2001, IAU Circ., 7772, 2

Pian, E., et al. 2000, ApJ, 536, 778

Podsiadlowski, P. 1992, PASP, 104, 717

Podsiadlowski, P., Joss, P. C., \& Hsu, J. J. L. 1992, ApJ, 391, 246

Pooley, D., \& Lewin, W. H. G. 2003, IAU Circ., 8110, 2 2004, IAU Circ., 8323, 2

Predehl, P., \& Schmitt, J. H. M. M. 1995, A\&A, 293, 889

Ramirez-Ruiz, E., García-Segura, G., Salmonson, J. D., \& Pérez-Rendón, B. 2005, ApJ, 631, 435

Readhead, A. C. S. 1994, ApJ, 426, 51

Rosenberg, I. 1970, MNRAS, 151, 109

Ryder, S. D., Murrowood, C. E., \& Stathakis, R. A. 2006, MNRAS, 369, L32

Ryder, S. D., Sadler, E. M., Subrahmanyan, R., Weiler, K. W., Panagia, N., \& Stockdale, C. 2004, MNRAS, 349, 1093

Schlegel, D. J., Finkbeiner, D. P., \& Davis, M. 1998, ApJ, 500, 525

Schlegel, E. M., Ryder, S., Staveley-Smith, L., Petre, R., Colbert, E., Dopita, M., \& Campbell-Wilson, D. 1999, AJ, 118, 2689

Smartt, S. J., Vreeswijk, P. M., Ramirez-Ruiz, E., Gilmore, G. F., Meikle, W. P. S., Ferguson, A. M. N., \& Knapen, J. H. 2002, ApJ, 572, L147

Soderberg, A. M., Gal-Yam, A., \& Kulkarni, S. R. 2004, GRB Circ., 2586, 1

Soderberg, A. M., Kulkarni, S. R., Berger, E., Chevalier, R. A., Frail, D. A., Fox, D. B., \& Walker, R. C. 2005a, ApJ, 621, 908

Soderberg, A. M., Nakar, E., Berger, E., \& Kulkarni, S. R. 2006, ApJ, 638, 930

Soderberg, A. M., et al. 2005b, ApJ, 627, 877

Stanek, K. Z., et al. 2003, ApJ, 591, L17

Stockdale, C. J., Maddox, L. A., Cowan, J. J., Prestwich, A., Kilgard, R., Immler, S., \& Krauss, M. 2006, AJ, 131, 889

Stockdale, C. J., Van Dyk, S. D., Sramek, R. A., Weiler, K. W., Panagia, N., Rupen, M. P., \& Paczynski, B. 2004, IAU Circ., 8282, 2

Sutaria, F. K., Chandra, P., Bhatnagar, S., \& Ray, A. 2003, A\&A, 397, 1011

Tuthill, P. G., Monnier, J. D., \& Danchi, W. C. 1999, Nature, 398, 487

van Dyk, S. D., Weiler, K. W., Sramek, R. A., Rupen, M. P., \& Panagia, N. 1994, ApJ, 432, L115

Waxman, E. 2004, ApJ, 602, 886

Weiler, K. W., Panagia, N., \& Montes, M. J. 2001, ApJ, 562, 670

Weiler, K. W., Panagia, N., \& Sramek, R. A. 1990, ApJ, 364, 611

Weiler, K. W., Sramek, R. A., Panagia, N., van der Hulst, J. M., \& Salvati, M. 1986, ApJ, 301, 790

Weiler, K. W., Sramek, R. A., van der Hulst, J. M., \& Panagia, N. 1982, in Proc. Adv. Study Inst., Supernovae: A Survey of Current Research (Dordrecht: Reidel), 281

Weiler, K. W., van Dyk, S. D., Discenna, J. L., Panagia, N., \& Sramek, R. A. 1991, ApJ, 380, 161

Weiler, K. W., van Dyk, S. D., Pringle, J. E., \& Panagia, N. 1992, ApJ, 399, 672

Wheeler, J. C., \& Levreault, R. 1985, ApJ, 294, L17

Williams, C. L., Panagia, N., Van Dyk, S. D., Lacey, C. K., Weiler, K. W., \& Sramek, R. A. 2002, ApJ, 581, 396

Wood-Vasey, W. M., \& Chassagne, R. 2003, IAU Circ., 8082, 1

Woosley, S. E., Eastman, R. G., \& Schmidt, B. P. 1999, ApJ, 516, 788 